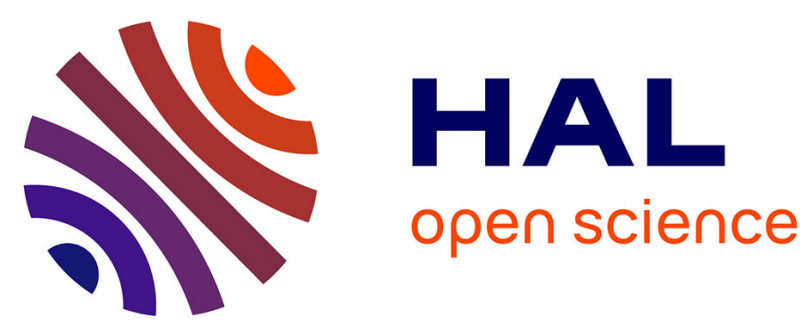

\title{
The gp27-like Hub of VgrG Serves as Adaptor to Promote Hcp Tube Assembly
}

\author{
Melvin G Renault, Jordi Zamarreño Beas, Badreddine Douzi, Maïalène \\ Chabalier, Abdelrahim Zoued, Yannick R Brunet, Christian Cambillau, Laure \\ Journet, E. Cascales
}

\section{To cite this version:}

Melvin G Renault, Jordi Zamarreño Beas, Badreddine Douzi, Maïalène Chabalier, Abdelrahim Zoued, et al.. The gp27-like Hub of VgrG Serves as Adaptor to Promote Hcp Tube Assembly. Journal of Molecular Biology, 2018, 430 (18 Pt B), pp.3143-3156. 10.1016/j.jmb.2018.07.018 . hal-01847145

\section{HAL Id: hal-01847145 \\ https://hal-amu.archives-ouvertes.fr/hal-01847145}

Submitted on 23 Jul 2018

HAL is a multi-disciplinary open access archive for the deposit and dissemination of scientific research documents, whether they are published or not. The documents may come from teaching and research institutions in France or abroad, or from public or private research centers.
L'archive ouverte pluridisciplinaire HAL, est destinée au dépôt et à la diffusion de documents scientifiques de niveau recherche, publiés ou non, émanant des établissements d'enseignement et de recherche français ou étrangers, des laboratoires publics ou privés. 
3 Melvin G. Renault ${ }^{1, \#}$, Jordi Zamarreño Beas ${ }^{1, \dagger, \#}$, Badreddine Douzi ${ }^{2, \dagger}$, Maïalène Chabalier ${ }^{1}$, 4 Abdelrahim Zoued ${ }^{1, \uparrow}$, Yannick R. Brunet ${ }^{1, \S}$, Christian Cambillau ${ }^{2}$, Laure Journet ${ }^{1}$, and Eric

Running head: T6SS hub-tube interaction.

8

${ }^{1}$ Laboratoire d'Ingénierie des Systèmes Macromoléculaires (LISM), Institut de Microbiologie de la 12 Méditerranée (IMM), Aix-Marseille Univ - Centre National de la Recherche Scientifique (CNRS), 13 UMR7255, 31 chemin Joseph Aiguier, 13402 Marseille Cedex 20, France.

$14{ }^{2}$ Architecture et Fonction des Macromolécules Biologiques (AFMB), Aix-Marseille Univ - Centre 15 National de la Recherche Scientifique (CNRS), UMR 7257, Campus de Luminy, Case 932, 13288 16 Marseille Cedex 09, France

† Current address: Laboratoire de Chimie Bactérienne (LCB), Institut de Microbiologie de la Méditerranée (IMM), Aix-Marseille Univ - Centre National de la Recherche Scientifique (CNRS), UMR7283, 31 chemin Joseph Aiguier, 13402 Marseille Cedex 20, France.

21 "Current address: Howard Hughes Medical Institute, Brigham and Women's Hospital, Division of 22 Infectious Diseases and Harvard Medical School, Department of Microbiology and Immunobiology, 23 Boston, Massachusetts, USA.

$24 \S$ Current address: Department of Microbiology and Immunobiology, Harvard Medical School, 77 25 Avenue Louis Pasteur, Boston, MA, 02115 USA.

26 \# These authors contributed equally to the work

$27 *$ To whom correspondence should be addressed. E-mail: cascales@imm.cnrs.fr 


\section{ABSTRACT}

3 Contractile injection systems are multiprotein complexes that use a spring-like

4 mechanism to deliver effectors into target cells. In addition to using a conserved

5 mechanism, these complexes share a common core known as the tail. The tail comprises

6 an inner tube tipped by a spike, wrapped by a contractile sheath, and assembled onto a

7 baseplate. Here, using the Type VI secretion system (T6SS) as a model of contractile

8 injection systems, we provide molecular details on the interaction between the inner

9 tube and the spike. Reconstitution into the $E$. coli heterologous host in absence of other

10 T6SS components and in vitro experiments demonstrated that the Hcp tube component

11 and the VgrG spike interact directly. VgrG deletion studies coupled to functional assays

12 showed that the $\mathrm{N}$-terminal domain of $\mathrm{VgrG}$ is sufficient to interact with Hcp, to initiate

13 proper Hcp tube polymerization, and to promote sheath dynamics and Hcp release. The

14 interaction interface between Hcp and VgrG was then mapped using docking

15 simulations, mutagenesis and cysteine-mediated cross-links. Based on these results, we

16 propose a model in which the VgrG base serves as adaptor to recruit the first Hcp

17 hexamer and initiates inner tube polymerization. 


\section{INTRODUCTION}

2 The Type VI secretion system (T6SS) is a multiprotein machine, widespread in Proteobacteria

3 and responsible for the delivery of effectors into prokaryotic as well as eukaryotic target cells,

4 thus participating to interbacterial competition and pathogenesis [1-8]. The T6SS comprises a

5 trans-envelope complex constituted of the TssJ, TssL and TssM proteins [9-11]. This TssJLM

6 membrane complex serves as a docking station for a tail-like structure [11-15]. The T6SS tail-

$7 \quad$ like structure is structurally, evolutionarily and functionally related to the tail of contractile

8 injection systems (CIS), a broad family of machines that use a spring-like mechanism to

9 deliver macromolecules into target cells [16-21]. A number of CIS act as autonomous

10 machineries and are released in the external medium where they bind to specific receptors

11 located at the cell surface of target cells. These include the prototypical contractile bacteriophages of the Myoviridae family that injects their genome into bacterial host cells, and particles such as R-pyocins, anti-feeding prophages and Photorhabdus virulence cassettes

14 [22-25]. Other CIS act intracellularly and are oriented toward the cell exterior. These include the Pseudoalteromonas luteoviolacea metamorphosis-associated and Amoebophilus asiaticus contractile machines, as well as the T6SS [16, 19, 20, 26-28]. These CIS are responsible for

17 the delivery of effector proteins that stimulate the genetic program of eukaryotic cells to induce morphogenesis, interfere with the cytoskeleton or the signalling pathway in eukaryotic cells, or cause cell damages in bacterial cells $[5,6,20,26,29,30]$. Hence, CIS participate to establishment of symbiosis, pathogenesis, modification of the host or bacterial competition.

21 The mechanism of action is conserved between all these machines: a needle is propelled by the contraction of a sheath and penetrates into the target cell to deliver macromolecules [28]. However, these systems have evolved additional modules to fulfil specific functions [21].

24 Extracellular CIS have fibers that mediate contact with the target cell by binding to specific 25 receptors. Intracellular CIS are anchored to the cell envelope by a membrane anchor or 
1 membrane complex that orients the CIS toward the exterior and serves as channel for the 2 passage of the needle during sheath contraction [21]. At the molecular level, the CIS is

3 constituted of a needle surrounded by a contractile sheath and built on an assembly platform, 4 the baseplate $[12,22,31,32]$. The needle is composed of an inner tube tipped by a puncturing 5 device $[19,22,28]$. The baseplate comprises conserved components that form wedges that 6 assemble around the central hub [12, 31, 33]. In most cases, the central hub connects the inner 7 tube with the puncturing device [34-36]. However, in T6SSs, the central hub and the 8 puncturing device are fused as a single polypeptide, a chimera protein called $\operatorname{VgrG}[1,37,38]$.

9 Hence it is predicted that $\operatorname{VgrG}$ represents both the central part of the baseplate and the tail 10 tube-puncturing device connector [31, 39, 40]. Indeed, interactions of VgrG with the inner 11 tube Hcp protein has been evidenced in Agrobacterium tumefaciens and with several baseplate components including TssK and the TssFG complex in enteroaggregative Escherichia coli (EAEC) [12, 41, 42]. The structure of the uropathogenic E. coli VgrG Nterminal fragment corresponding to the hub has been solved [38], as well as the full-length VgrG1 protein from Pseudomonas aeruginosa [43]. VgrG form trimers and is constituted of several domains: (i) an N-terminal domain that resembles the bacteriophage T4 gp27 and other phages hub proteins and that has, in the trimer, the overall structure of two superimposed disks, (ii) an oligonucleotide/oligosaccharide-binding (OB)-fold domain which is structurally similar to the bacteriophage T4 gp5 OB-fold domain, and (iii) the puncturing device which is comparable to the membrane-penetrating gp5 triple-stranded $\beta$-helix $[35,37$, $38,43-45]$. In addition to the core gp27-gp5 structure, additional domains could be found at the VgrG C-terminal extremity, such as effector domains bearing an enzymatic activity or adaptor domains that binds specific effectors $[6,7,37,46]$. Therefore, T6SS-associated VgrG proteins have a modular architecture and serve as hub for baseplate assembly, syringe for target cell membrane penetration, docking site for effector translocation, and connector for 
1 tail tube. In T6SS, the tail polymerizes once the baseplate docks to the membrane complex

$2[11,12]$. Assembly of the inner tube proceeds by the addition of Hcp hexameric rings that

3 stack on each other in a head-to-tail organization [47]. Hcp shares the same fold as tailed

4 phages major tail proteins that are structurally related to the upper ring domain of the phage

5 T4 gp27 hub [38, 44, 48, 49]. Polymerization of the sheath proceeds by the addition of TssBC

6 complexes at the distal end of the growing tail structure [19, 28, 50-52]. The assembly of the

7 tube is coordinated with that of the sheath: in absence of sheath, the Hcp hexameric rings do

8 not stack properly whereas no sheath extension occurs in absence of Hcp [47]. The

9 coordinated polymerization of the tail is mediated by TssA, a protein that associates to the

10 baseplate and then recruits and incorporates new tube and sheath blocks at the distal extremity

$11[53,54]$. However, it is not yet known how the first ring of Hcp is recruited to the baseplate and how Hcp interacts with VgrG in the final needle structure. Interestingly, the N-terminal disk of VgrG has a structure comparable to that of the hexameric Hcp, and therefore has been proposed to connect the axial 6-fold symmetry of Hcp with the 3-fold axial symmetry of the $\beta$-helix needle $[38,44]$. VgrG therefore constitutes a good candidate to serve as nucleation factor for the inner tube. Here, bacterial two-hybrid (BACTH) and co-immunoprecipitation (co-IP) experiments show that VgrG1 interacts with Hcp1 in EAEC. Using shorter variants of VgrG1, we provide evidence that the gp27-like hub domain of VgrG1 is sufficient to interact with Hcp1, and that the strength of this interaction is comparable to that between Hcp1 hexamers. We then demonstrate that the VgrG hub domain is sufficient for the assembly and the mechanism of action of the T6SS, as it promotes assembly of the inner tube and of the contractile sheath, and permits Hcp1 release in the supernatant. Finally, targeted mutagenesis and cysteine cross-linking studies reveal that the base of the VgrG1 gp27-like hub interacts with the head surface of Hcp1. Taken together, these results allow to propose a model for the assembly and the architecture of the hub/tube interface. 


\section{RESULTS}

\section{VgrG1 interacts with Hcp1.}

To test whether VgrG and Hcp interact, the $\operatorname{vgrG}$ and $h c p$ genes of the EAEC sci-1 gene cluster (vgrG1, EC042_4533, accession number (GI): 284924254; hcp1, EC042_4529, GI: 284924250) were cloned into BACTH vectors, in frame with the T18 and T25 domains of the Bordetella adenylate cyclase, respectively. The results shown on reporter plates demonstrate that Hcp1 and VgrG1 interact (Fig. 1A). The interaction between the two proteins was confirmed by co-immunoprecipitation (co-IP) in the heterologous host E. coli K-12: VSV-Gtagged VgrG1 specifically co-immunoprecipitated with FLAG-tagged Hcp1 (Fig. 1B and 1C). These results show that the two proteins interact, and that this interaction is likely to be direct because it occurs in the absence of the other T6SS genes in E. coli K-12.

\section{The VgrG1 N-terminal gp27-like hub is sufficient to mediate interaction with Hcp1.}

VgrG proteins form trimers with a modular architecture, as a result of fusion events between gp27-like, gp5-like and adaptor/effector domains. In EAEC, the Sci-1 VgrG protein, VgrG1, is predicted to adopt a classic VgrG fold with the gp27-like hub and gp5 OB-fold and $\beta$-helix domains. In addition, EAEC VgrG1 carries a C-terminal extension, comprising DUF2345 and TTR domains, which binds to the Tle1 phospholipase effector [55]. To determine the region of VgrG1 that interacts with Hcp1, we conducted a deletion mutagenesis coupled to co-IP assays. First, VgrG1 boundaries were defined by modelling its structure based on the structure of the P. aeruginosa VgrG1 protein (PDB: 4UHV; [43]): gp27-like hub (residues 1-386), gp5 
1 OB-fold (residues 387-490), gp5 $\beta$-helix (residues 491-573), and C-terminal extension

2 (residues 574-841) (Fig. 2A and 2B). Fragments corresponding to the gp27-OB-gp5-C

3 ( $\mathrm{DDUF}), \mathrm{gp} 27-\mathrm{OB}(\Delta \mathrm{gp} 5)$ and gp27 $(\Delta \mathrm{OB})$ regions (Fig. $2 \mathrm{~B})$ were fused to the VSV-G

4 epitope, and the interaction of the VgrG truncated variants with Hcp1 was assayed using co-

5 IP. Fig. 2C shows that none of the deletions affects the interaction with Hcp1, and hence that

6 the gp27-like hub domain is sufficient to promote VgrG1-Hcp1 complex formation. To

7 quantify the strength of this association, VgrG1- $\Delta \mathrm{OB}$ and Hcpl were purified. However,

8 while the isolated gp27 domain of VgrG1 was not stable, a variant comprising the OB fold

9 (i.e., VgrG1- $\Delta \mathrm{gp} 5$ ) was purified to homogeneity (Fig. 2D, left upper panel). The VgrG1- $\Delta g p 5$

10 interaction with Hcp1 was recorded by microscale thermophoresis (MST). VgrG1- $\Delta$ gp5 was

11 labelled with the amine-reactive blue fluorescent NHS dye and titrated against increasing

12 concentrations of Hcpl (Fig. 2D, right upper panel). The fitted traces confirmed that VgrG1-

$13 \Delta \mathrm{gp} 5$ interacts with Hcp1 with a $K_{\mathrm{D}}$ value of $\sim 2.8 \mu \mathrm{M}$ (Fig. 2D, lower panel). Interestingly,

14 the VgrG-Hcp association is not restricted to the EAEC Sci-1 T6SS, as MST assays demonstrated that (i) Hcp2, the Hcp protein encoded by the second T6SS gene cluster in the EAEC 17-2 strain, interacts with its cognate VgrG, VgrG2- $\Delta$ gp5, with a $K_{\mathrm{D}}$ of $\sim 9.4 \mu \mathrm{M}$ (Fig.

$17 \mathrm{~S} 1 \mathrm{~A})$, and (ii) the $V$. cholerae $\mathrm{Hcp}\left(\mathrm{Hcp}_{\mathrm{Vc}_{\mathrm{c}}}\right)$ and $\operatorname{VgrG}\left(\mathrm{VgrG}_{\mathrm{V}_{\mathrm{c}}}\right)$ interact with a $K_{\mathrm{D}}$ of $\sim 4.1$ $\mu \mathrm{M}$ (Fig. S1B). Hence, VgrG proteins interact with cognate Hcp proteins with an affinity in

19 the 1-10 $\mu \mathrm{M}$ range. To gain further information about the specificity of Hcp protein towards VgrG, we performed cross-experiments where we tested the interaction of Hcp1 and Hcp2

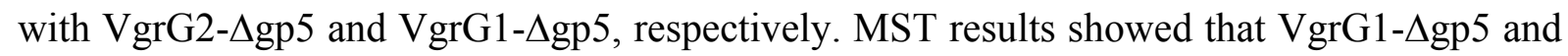
Hcp2 $\left(K_{\mathrm{D}} \sim 35.5 \mu \mathrm{M}\right.$; Fig. S1C), and VgrG2- $\Delta$ gp5 and Hcp1 $\left(K_{\mathrm{D}} \sim 22.4 \mu \mathrm{M}\right.$; Fig. S1D $)$ interact, although with lower affinity compared to the VgrG1- $\Delta$ gp5-Hcp1 and VgrG2- $\Delta$ gp5Hcp2 complexes. These results suggest that VgrG and Hcp proteins interact but that the affinity is significantly higher between cognate pairs. However, in vivo interbacterial 
1 competition assays showed that VgrG2 and Hcp2 are unable to compensate for the absence of

$2 \operatorname{vgrG1}$ and $h c p 1$, respectively (Fig. S2). It is therefore unlikely that non-cognate $\mathrm{VgrG}$ and

3 Hcp proteins work together in EAEC cells.

4

5 The VgrG1 gp27-like hub is sufficient to promote Hcp1 tube assembly, sheath dynamics and Hcp1 release in the medium.

7 T6SS biogenesis starts with the formation of the membrane complex and the recruitment of

8 the baseplate prior to coordinated assembly of the tail tube and sheath $[11,12,53,56]$. Once

9 assembled, the T6SS sheath contracts and propels the Hcp tube toward the target cell to deliver effectors [57]. A recent study has demonstrated that EAEC T6SS-mediated killing requires the full-length VgrG protein, which comprises the C-terminal extension that binds

12 the Tle1 phospholipase effector [55]. However no information is available regarding the 13 contribution of VgrG1 domains for T6SS biogenesis. We thus tested whether VgrG1- $\triangle \mathrm{OB}$ is 14 sufficient to promote tail tube and tail sheath assembly, and Hcpl release in the medium (Fig. 3).

It has been proposed that assembly of the tail tube and sheath is concomitant, the 17 addition of a new Hcp ring immediately preceding the polymerization of a TssBC sheath 18 strand [47]. The arrangements of Hcp rings could be probed by disulfide bond formation 19 between cysteine side-chains located on each side of the Hcp ring [47, 58]. In vivo, Hcp1 20 rings organize in a head-to-tail manner with an estimated $K_{\mathrm{D}}$ of $7 \mu \mathrm{M}$ to form the tail tube 21 [59]. This organization is controlled by components of the baseplate, including VgrG1: in 22 their absence, Hcp1 rings associate randomly in head-to-tail, head-to-head and tail-to-tail arrangements $[12,47]$. We thus tested the minimal VgrG1 requirements for proper Hcp1 tube 
1 assembly. Formation of disulfide bonds between pairs of cysteines that specifically report

2 head-to-head, tail-to-tail and head-to-tail Hcp1 assembly was tested in cells producing the

3 full-length VgrG1 or VgrG1 gp27-like hub (VgrG1- $\Delta \mathrm{OB})$. Cysteine-mediated cross-linking in

4 oxidative conditions showed that $\operatorname{VgrG} 1-\Delta \mathrm{OB}$ is sufficient to control proper assembly of

5 Hcp1 tube exclusively in head-to-tail arrangements (Fig. 3A). Furthermore, dynamics of the

6 tail sheath could be observed by fluorescence microscopy using fluorescently-labelled TssB.

7 Time-lapse recordings show that the VgrG1- $\Delta \mathrm{OB}$ construct is sufficient to mediate extension

8 and contraction of the T6SS tail sheath (Fig. 3B and Fig. S3). These results suggest that the

9 VgrG1 gp27-like hub is sufficient for proper assembly and action of the EAEC Sci-1 T6SS.

10 This hypothesis was confirmed by the observation that VgrG1- $\Delta \mathrm{OB}$ is sufficient to enable

11 Hcp1 release in the milieu, a reporter of sheath contraction (Fig. 3C). The ability of VgrG1-

$12 \Delta \mathrm{OB}$ to promote T6SS assembly is not due to the formation of functional heterologous

$13 \mathrm{VgrG} 2 / \mathrm{VgrG} 1-\Delta \mathrm{OB}$ trimer assemblies as production of VgrG1- $\Delta \mathrm{OB}$ in $\operatorname{vgr}$ G1-vgrG2 mutant

14 cells is sufficient to support Hcp release in the culture supernatant (Fig. S4).

The head side of Hcp1 interacts with VgrG1.

To define the side of Hcp1 that contacts VgrG1, we used tryptophan substitution variants of Hcpl previously described to prevent assembly of Hcp tubes by sterically perturbing Hcp hexameric rings association [47, 59]. These tryptophan substitutions were positioned at the head (S158W) or tail (N93W) side of Hcp1. Bacterial two-hybrid assays showed that introduction of a tryptophan residue at position Ser-158 disrupts the interaction with VgrG1 whereas a tryptophan at position Asn-93 does not impact Hcp1-VgrG1 complex formation (Fig. 4A). The strong impact of the S158W mutation on VgrG1-Hcp1 complex

24 formation was further confirmed by co-immunoprecipitation (Fig. 4B). This result suggests 
1 that the head side of Hcp1 contacts VgrG1 (Fig. 4C). The current model of EAEC T6SS

2 assembly proposes that TssA controls T6SS tail polymerization and binds to the distal end of

3 the tube/sheath during extension $[53,54]$. This model implies that TssA should contact Hcp1

4 at the opposite side relative to $\operatorname{VgrG1}$ (i.e., the tail side). Bacterial two-hybrid experiments

5 using TssA and the Hcp1 tryptophan variants mirrored the VgrG1-Hcp1 interaction: the Hcp1

6 N93W variant does not interact with TssA whereas a tryptophan at position 158 does not

7 affect Hcp1-TssA interaction (Fig. 4A and 4B). Taken together, these results indicate that

8 TssA and VgrG1 bind to the two opposite sides of Hcp1: VgrG1 contacts the head side

9 whereas TssA interacts with the tail side (Fig. 4C).

The VgrG1 loop 360-362 is required for VgrG1-Hcp1 complex formation.

Because Hcp1 likely interacts with the basal side of the VgrG1 gp27-like domain, we engineered a VgrG1 variant in which one of the protruding loops (Fig. 4C), comprising residues Ser-360, Arg-361 and Lys-362, was exchanged by three glycine residues (S360GR361G-K362G; hereafter called $\left.\operatorname{VgrG}_{1} 1_{\text {loop}}\right)$. These mutations had no impact on VgrG1 multimer formation, as monitored by bacterial two-hybrid (Fig. 4D). However, proteinprotein interaction assays demonstrated that the VgrG1 360-362 region is important for VgrG1-Hcp1 complex formation, as no interaction was detected between Hcp1 and VgrG1 $1_{\text {loop }}$ by bacterial two-hybrid and co-immunoprecipitation (Fig. 4B and 4D). Finally, production of the $\operatorname{VgrG1} 1_{\text {loop }}$ variant does not compensate the absence of VgrG1 for in vivo Hcp1 tube formation (Fig. 4E) and inter-bacterial activity (Fig. 4F). These results demonstrate that the base of the VgrG1 hub-like domain, or at least the 360-362 loop, is critical for mediating 23 functional contacts with Hcp1. 
2 Disulfide bond formation identifies the T6SS hub-tube interface.

To gain detailed insights onto the VgrG1-Hcp1 interface, we introduced cysteine

4 residues at the base of the VgrG1 gp27 hub domain and at each side of the Hcp1 hexamer. We

5 reasoned that the thiol groups of cysteine side-chains would form disulfide bonds if located at

6 appropriate distances $(\mathrm{C} \alpha-\mathrm{C} \alpha$ distance $<7 \AA)$. We first performed molecular docking

7 simulations using the structure of Hcp1 [59] and the generated structural model of VgrG1.

8 Based on the best matches, cysteine residues were rationally positioned in loops, substituting

9 residues sharing a 3 to $7-\AA \AA C \alpha-C \alpha$ distance. Six positions were substituted on the cysteineless (C38S) Hcp1: Gln-24, Asn-46, Gly-48, Ala-95, Gly-96 and Ser-158. Gln-24, Ala-95 and

11 Gly-96 locate at the Hcp1 tail side whereas Asn-46, Gly-48 and Ser-158 locate at the Hcp1 12 head side. The Hcp1 Q24C, G48C, A95C, G96C, and S158C mutants were previously shown 13 to be functional [47]. The VgrG1 protein possesses five native cysteine residues, including two in the gp27-like hub domain (Cys-27 and Cys-379). Because the bacterial cytoplasm is considered as reducing, formation of disulfide bonds between the VgrG1 and Hcp1 cysteine variants was catalyzed in presence of the oxidative agent copper(II) orthophenanthroline. Fig. 5A shows that none of the VgrG1 native cysteines engages in disulfide bond formation with the Hcp1 cysteine variants. Thus, two residues located in the VgrG1 360-362 loop that is important for VgrG1-Hcp1 complex formation were substituted by cysteines in the wild-type VgrG1 protein: Arg-361 and Lys-362. Interbacterial competition assays demonstrated that the Hcp1 N46C and VgrG1 R361C and K362C mutants are functional (Fig. S5). Fig. 5B shows that higher molecular weight species (denoted with * and ** in the anti-VSV-G and antiFLAG immunoblots, respectively) are observable for four combinations involving VgrG1 
1 species $(\sim 120 \mathrm{kDa})$ is compatible with a complex between $\operatorname{VgrG} 1$ (theoretical weight: 93

$2 \mathrm{kDa}$ ) and Hcp1 (theoretical weight: $18 \mathrm{kDa}$ ). These complexes are likely to be covalently

3 bound by disulfide bridges as they dissociate upon addition of a reducing agent (Fig. 5C), and

4 likely comprise both VgrG1 and Hcp1 as they are recognized by both the VSV-G and FLAG 5 antibodies (Fig. 5D).

\section{DISCUSSION}

In this study, BACTH, co-IP, MST and disulfide bond cross-linking assays

9 demonstrated that the component of the T6SS inner tube, Hcp1, interacts with the needle spike VgrG1 in EAEC. A complex comprising the Agrobacterium tumefaciens VgrG and Hcp proteins has been previously reported using co-immunoprecipitation [41]. Interaction between the VgrG spike and the Hcp tube component is likely to be conserved in all T6SS. Indeed, additional results demonstrated that the Hcp and VgrG proteins associated with the EAEC Sci-2 and $V$. cholerae T6SS gene clusters also interact. Interestingly, we also showed that cross-interactions between non-cognate Hcp and VgrG proteins from the two EAEC T6SS gene clusters exist, albeit with a significant decrease in affinity compared to cognate pairs.

17 However, in vivo studies showed that VgrG2 and Hcp2 cannot functionally replace VgrG1 18 and Hcp1, respectively.

Our VgrG1 deletion studies demonstrated that its gp27-like domain is sufficient to interact with Hcp1. Interestingly, we showed that neither the gp5 OB-fold nor the $\beta$-helix or

21 the C-domains are essential for the assembly for the T6SS apparatus since the gp27 domain is 22 sufficient to promote Hcp1 assembly, sheath extension and contraction, and Hcp1 release in the milieu. In addition, a previous report from our laboratory had shown that the VgrG1 C- 
1 terminal extension is necessary to cause target cell lysis by binding and delivering the Tle1

2 phospholipase effector [55]. Taken together, these information suggest that the VgrG1 gp27-

3 like hub is sufficient to promote assembly of the T6SS, and that the $\beta$-helix and C-terminal

4 extension are required to penetrate the cell envelope of the target cell and to deliver effectors.

5 The observation that Hcp1 is released in the medium of cells producing the VgrG1 gp27-like

6 domain further suggests that the VgrG1 $\beta$-helix is not required for piercing the outer

7 membrane of the producing cell, and therefore that the needle should cross the outer

8 membrane through a dedicated channel such as that proposed by a conformational change of

9 the outermost portion of the TssJLM complex [11]. Our data also showed that the VgrG1 OB-

10 fold domain is not necessary for promoting Hcp1 tube formation, which is in agreement with

11 the suggestion that the OB-fold acts as a symmetry connector between the six-fold gp27-like

12 hub domain and the three-fold $\beta$-helix needle domain [38].

Mutational studies and docking simulations coupled to disulfide bond assays detailed the contacts between Hcp1 and VgrG1 (Fig. 6). Using substitutions with tryptophan, a bulky side-chain aminoacid, we showed that the head side of the Hcp1 hexameric ring mediates contacts with the gp27-like base of VgrG1. In agreement with this organization, the opposite side of Hcp engages contacts with TssA, a subunit that coordinates the assembly of the inner tube with the polymerization of the sheath and that remains associated with the distal end of the tail during its extension [53]. Formation of disulfide bonds between cysteine residues located at positions 46 and 48 of Hcp1 and 361 and 362 of VgrG1 allows to propose a model 21 for Hcp1 binding to VgrG1 (Fig. 6).

Interestingly, Leiman et al. noted that the gp27-like domain of the VgrG trimer has a fold similar to that of an Hcp hexamer [38]. When superimposed, the VgrG basal side corresponds to the tail side of Hcp. By interacting with the Hcp1 head side, VgrG1 engages 
1 into a head-to-tail organization with Hcp1, and hence in an association comparable to that

2 between two Hcp1 hexamers. We thus propose that the hub formed by the VgrG gp27-like

3 domain trimer serves as an adaptor to recruit the first Hcp hexameric ring and thus initiates

4 the assembly of the inner tube. In agreement with this proposal, in vivo tube formation assays

5 have shown that the Hcp1 tube does not assemble properly in absence of vgrG1 [47].

6 Interestingly, affinity measurements demonstrate that Hcp proteins interact with their cognate

7 VgrG with a $\mathrm{K}_{D}$ in the 1-10 micromolar range. With a $\mathrm{K}_{D}$ of $\sim 3 \mu \mathrm{M}$, the affinity of Hcp1 for

8 VgrG1 is comparable - albeit better - to Hcp1 hexamers association (7 $\mu \mathrm{M})$ [59]. Based on the

9 slightly better affinity for VgrG1, we propose that an Hcp1 hexamer binds to the free base of

10 VgrG1, which then becomes unavailable. Hcp1 tube polymerization can then proceed by the

11 addition of new Hcp1 hexamers on each other at the distal end of the growing structure (Fig.

12 7). This model agrees with recent data showing that the tail extends by the distal end $[51,53]$

\section{MATERIAL AND METHODS}

Bacterial strains, medium, and growth conditions. Strains used in this study are listed in Table S1. Escherichia coli DH5a (New England Biolabs) was used for cloning procedures, W3110 for coimmunoprecipitations, BTH101 [60] for bacterial two-hybrid assays and BL21(DE3)pLys (Invitrogen) for protein purification. The enteroaggregative E. coli (EAEC) 17-2 strain and its $\Delta \operatorname{vgr} G 1, \Delta h c p 1$, $\Delta v g r G 1 \Delta h c p 1$, and $\Delta v g r G 1$-tssB-mCh derivatives [12] have been used for in vivo studies. Cells were routinely grown in Lysogeny broth $(\mathrm{LB})$ at $30^{\circ} \mathrm{C}$ or $37^{\circ} \mathrm{C}$. sci-1 gene expression was induced in SIM (Sci-1 inducing medium; M9 minimal medium supplemented with LB $10 \%$, glycerol $0.4 \%$, casaminoacids $40 \mu \mathrm{g} \cdot \mathrm{mL}^{-1}, \mathrm{MgCl}_{2} 2 \mathrm{mM}, \mathrm{CaCl}_{2} 0.1 \mathrm{mM}$, Vitamin B1 $200 \mu \mathrm{g} \cdot \mathrm{mL}^{-1}$ ) [61]. Terrific broth (TB; $1.2 \%$ peptone, $2.4 \%$ yeast extract, $72 \mathrm{mM} \mathrm{K}_{2} \mathrm{HPO}_{4}, 17 \mathrm{mM} \mathrm{KH}_{2} \mathrm{PO}_{4}$, and $0.4 \%$ glycerol) was used for protein overproduction. Plasmids were maintained by addition of ampicillin $\left(100 \mu \mathrm{g} . \mathrm{mL}^{-1}\right)$, kanamycin $\left(50 \mu \mathrm{g} \cdot \mathrm{mL}^{-1}\right.$ for E. coli $\mathrm{K}-12 ; 100 \mu \mathrm{g} \cdot \mathrm{mL}^{-1}$ for EAEC) or chloramphenicol $\left(40 \mu \mathrm{g} \cdot \mathrm{mL}^{-1}\right)$. 
1 Gene expression was induced with 0.1-0.5 mM isopropyl- $\beta$-thio-galactoside (IPTG) or L-arabinose $2(0.02 \%)$.

3 Plasmid construction. Plasmids used in this study are listed in Table S1. Polymerase Chain Reactions 4 (PCR) were performed using a Biometra thermocycler using Pfu Turbo DNA polymerase (Agilent 5 Technology). Custom oligonucleotides, listed in Table S1, were synthesized by Sigma Aldrich. EAEC 17-2 chromosomal DNA was used as a template for all PCRs. E. coli strain DH5 $\alpha$ was used for cloning procedures. Bacterial two-hybrid plasmids encoding T18 and T25 fusions to Hcp1 and VgrG1, plasmid pBAD-VgrG1 $\mathrm{V}$, encoding C-terminally VSV-G-tagged VgrG1 from the pBAD33 vector, plasmids pUC-Hcp $1_{\mathrm{F}}$ and pUC-Hcp2 $2_{\mathrm{F}}$, encoding N-terminally FLAG-tagged Hcp1 and Hcp2 from the pUC12 vector, and plasmids pDest14-Hcp1 and pDest14-Hcp2, encoding Hcp1 and Hcp2 fused to a C-terminal $6 \times$ His tag have been previously described $[10,42,59,62]$. Plasmids encoding truncated fragments of VgrG have been constructed by restriction/ligation-free cloning, as previously described $[62,63]$. Briefly, fragments of interest were amplified with oligonucleotides introducing extensions annealing to the target vector. The double-stranded product of the first PCR has then been used as oligonucleotides for a second PCR using the target vector as template. Except the N46C variant, cysteine and tryptophan variants of Hcp1 have been previously described [47, 59]. Hcp1 N46C and VgrG1 R361C, K362C and S360G-R361G-K362G (VgrG1 $1_{\text {loop }}$ ) substitutions have been introduced by site-directed mutagenesis using complementary pairs of oligonucleotides and the Pfu Turbo high fidelity polymerase (Agilent technologies). For protein purification, the DNA sequences encoding the

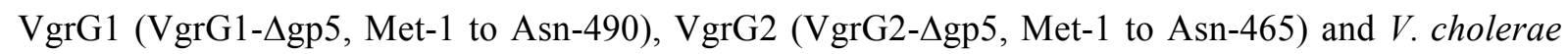
strain 0395 VgrG (VgrG $\mathrm{Vc}_{\mathrm{Vc}}-\Delta \mathrm{gp} 5$, Met-1 to Gln-471) gp27/OB regions were amplified from EAEC 172 or $V$. cholerae $\mathrm{O} 395$ and cloned into the pETG20A expression vector using standard Gateway procedures to yield pETG20A-VgrG1- $\Delta \mathrm{gp} 5$, pETG20A-VgrG2- $\Delta \mathrm{gp} 5$, and pETG20A-VgrG $\mathrm{Vc}_{\mathrm{c}}-\Delta \mathrm{gp} 5$, respectively. The resulting constructions allow the production of VgrG1- $\Delta \mathrm{gp} 5, \operatorname{VgrG} 2-\Delta \mathrm{gp} 5$, and $\operatorname{VgrG}_{\mathrm{Vc}}-\Delta \mathrm{gp} 5$ fused to an N-terminal thioredoxin followed by a $6 \times$ His tag and a Tobacco Etch Virus 
1 and cloned into the pDEST14 vector to yield pDest14-Hcp $\mathrm{vc}_{\mathrm{v}}$. All constructs have been verified by

2 DNA sequencing (Eurofins).

3 In vivo T6SS activity reporter assays. The interbacterial competition assay has been performed as

4 described [55]. The Hcp release assay was performed as described [62] except that cells were grown 5 in SIM to absorbance at $\lambda=600 \mathrm{~nm}\left(A_{600}\right)$ of 0.6. The periplasmic TolB marker was used as control for 6 cell integrity.

7 Fluorescence microscopy. Overnight cultures of EAEC 17-2 derivative strains were diluted 1:100 in 8 SIM medium and grown to an $A_{600} \sim 0.6$ to maximise the expression of the $s c i-1$ T6SS gene cluster 9 [61]. Cells were concentrated to an $A_{600}=5$ in SIM, spotted on a thin pad of $1.5 \%$ agarose in SIM and covered with a cover slip. Microscopy recordings and digital image processing have been performed as previously described $[42,47,53,64]$. The $\mathrm{Z}$ project (average intensity) plugin has been used to merge and flatten all Z-planes. Microscopy analyses were performed at least three times, each with 13 technical triplicate, and a representative experiment is shown.

14 Overproduction and purification of the EAEC Hcp and VgrG proteins. E. coli BL21(DE3) pLysS (Invitrogen) cells were transformed with the pDEST14 derivatives encoding Hcp proteins or pETG20A derivatives encoding VgrG gp27/OB domains. Overnight cultures grown in TB supplemented with ampicillin $\left(100 \mu \mathrm{g} \cdot \mathrm{mL}^{-1}\right)$ and chloramphenicol $\left(35 \mu \mathrm{g} \cdot \mathrm{mL}^{-1}\right)$ at $37^{\circ} \mathrm{C}$ were diluted in TB and grown at $37^{\circ} \mathrm{C}$ to $A_{600} \sim 0.6$. The temperature was then decreased to $25^{\circ} \mathrm{C}$ and the expression of the $h c p$ or $\operatorname{vgr} G$ genes was induced with $500 \mathrm{mM}$ IPTG for $18 \mathrm{~h}$. Cells were harvested, resuspended in buffer $\mathrm{A}(50 \mathrm{mM}$ Tris- $\mathrm{HCl}(\mathrm{pH} 8.0), 300 \mathrm{mM} \mathrm{NaCl})$ supplemented with $1 \mathrm{mM}$ of EDTA, $0.5 \mathrm{mg} \cdot \mathrm{mL}^{-1}$ of lysozyme, and phenylmethylsulfonyl fluoride (PMSF), submitted to three freeze-thawing cycles and sonicated after the addition of $20 \mu \mathrm{g} \cdot \mathrm{mL}^{-1}$ of DNase and $20 \mathrm{mM}$ of $\mathrm{MgCl}_{2}$. Insoluble material was discarded by centrifugation for $30 \mathrm{~min}$ at $16,000 \times g$. All the subsequent purification steps were performed using an AKTA FPLC system. For Hcp proteins, the soluble fraction was loaded onto a 5-mL HisTrap FF column (GE Health Sciences). After extensive washing, the Hcp proteins were eluted in one step gradient of Imidazole $250 \mathrm{mM}$ in Buffer A. For VgrG 
1 proteins, we followed the same protocol used for Hcp proteins with additional purification steps.

2 Briefly, the soluble proteins purified after the first HisTrap purification step were desalted on a HiPrep 3 26/10 column (Sephadex ${ }^{\mathrm{TM}}$ G-25, Amersham Biosciences), and VgrG proteins were obtained by 4 cleavage using $2 \mathrm{mg}$ of TEV protease for $18 \mathrm{~h}$ at $4{ }^{\circ} \mathrm{C}$ and collected in the flow-through of a 5-mL 5 HisTrap FF column. The resulting soluble proteins were concentrated using the centricon technology 6 (Millipore, 10-kDa cut-off). After concentration, the soluble Hcp or VgrG proteins were passed 7 through a Sephadex 200 26/60 column pre-equilibrated with $25 \mathrm{mM}$ Tris- $\mathrm{HCl}(\mathrm{pH} 7.5), 100 \mathrm{mM} \mathrm{NaCl}$.

8 Protein-protein interaction assays. Bordetella adenylate cyclase-based bacterial two-hybrid [42], co9 immuno-precipitation [13] and oxidative cross-linking [47] experiments have been performed as 10 previously described. Microscale thermophoresis experiments were performed using a Monolith 11 NT.115 apparatus (NanoTemper). VgrG1- $\Delta \mathrm{gp} 5, \operatorname{VgrG} 2-\Delta \mathrm{gp} 5$ and $\mathrm{VgrG}_{\mathrm{Vc}}-\Delta \mathrm{gp} 5$ were labeled with the blue-fluorescent dye NT-495-NHS (NanoTemper) and the buffer was exchanged for the assay buffer (25 mM Tris- $\mathrm{HCl}$ (pH 7.5), $200 \mathrm{mM} \mathrm{NaCl}$, glycerol 5\%, 0.05\% Tween-20) using a Nap5 column (GE

14 Healthcare). Titrations were conducted with a constant $200 \mathrm{nM}$ fluorophore-labeled VgrG protein 15 solution against up to $600 \mu \mathrm{M}$ of Hcp proteins. Each data point was measured in triplicate. Single-site 16 fitting was performed using the NanoTemper data analysis software.

Protein modeling and molecular docking simulations. The available structure of full-length VgrG1 protein from P. aeruginosa (PDB: 4MTK [Sycheva, Shneider and Leiman, unpublished] and 4UHV [43]; 25.5/58.4\% identity/similarity with EAEC VgrG1) was used as template to generate a model of the full-length VgrG1 from EAEC using the Homology modeling server accessible from the WHAT IF interactive interface. The HADDOCK2.2 webserver interface was used for molecular docking 22 simulations [65].

Miscellaneous. Sodium Dodecyl Sulfate-Poly Acrylamide Gel Electrophoresis (SDS-PAGE) was performed using standard protocols. For immunostaining, proteins were transferred onto nitrocellulose membranes, and immunoblots were probed with primary antibodies and goat secondary antibodies coupled to alkaline phosphatase and were developed in alkaline buffer in presence of 5-bromo-4- 
1 chloro-3-indolylphosphate and nitroblue tetrazolium. The anti-FLAG (clone M2, Sigma-Aldrich), and

2 anti-VSV-G (clone P5D4, Sigma-Aldrich) monoclonal antibodies, and alkaline phosphatase-

3 conjugated goat anti-mouse secondary antibodies (Beckman Coulter) have been purchased as

4 indicated and used as recommended by the manufacturer. The anti-TolB polyclonal antibody is from

5 our laboratory collection.

\section{ACKNOWLEDGEMENTS}

8 We thank the members of the Cascales, Cambillau, Lloubès, Bouveret and Sturgis research groups for 9 insightful discussions, and Moly Ba, Isabelle Bringer, Annick Brun and Olivier Uderso for technical 10 assistance. This work was supported by the Aix-Marseille Université (AMU), the Centre National de 11 la Recherche Scientifique (CNRS) and grants from the Agence Nationale de la Recherche to E.C. (ANR-10-JCJC-1303-03, ANR-14-CE14-0006-02 and ANR-17-CE11-0039-01). Undergraduate

13 laboratory works of M.G.R. and J.Z.B. were supported by the ANR-14-CE14-0006-02 and ANR-1014 JCJC-1303-03 grants, respectively. B.D. was supported by a Fondation pour la Recherche Médicale 15 (FRM) grant to C.C. (DEQ2011-0421282). A.Z. was supported by a doctoral fellowship from the 16 French Ministère de la Recherche and a FRM end-of-thesis fellowship (FDT20140931060). Y.R.B. 17 was supported by a doctoral fellowship from the French Ministère de la Recherche.

19 REFERENCES

20 [1] E. Cascales, The type VI secretion toolkit, EMBO Rep. 9 (2008) 735-741.

21 [2] J.M. Silverman, Y.R. Brunet, E. Cascales, J.D. Mougous, Structure and regulation of the type 22 VI secretion system, Ann. Rev. Microbiol. 66 (2012) 453-472.

23 [3] S.J. Coulthurst, The Type VI secretion system - a widespread and versatile cell targeting system. Res Microbiol. 164 (2013) 640-654. 
[4] N, Kapitein, A. Mogk, Deadly syringes: type VI secretion system activities in pathogenicity and interbacterial competition, Curr. Opin. Microbiol. 16 (2013) 52-58.

[5] A.B. Russell, S.B. Peterson, J.D. Mougous, Type VI secretion system effectors: poisons with a purpose, Nat. Rev. microbiol. 12 (2014) 137-148.

[6] E. Durand, C. Cambillau, E. Cascales, L. Journet, VgrG, Tae, Tle, and beyond: the versatile arsenal of type VI secretion effectors, Trends Microbiol. 22 (2014) 498-507.

J. Alcoforado Diniz, Y.C. Liu, S.J. Coulthurst, Molecular weaponry: diverse effectors delivered by the type VI secretion system, Cell. Microbiol. 17 (2015) 1742-1751.

F.R. Cianfanelli, L. Monlezun, S.J. Coulthurst, Aim, load, fire: the type VI secretion system, a bacterial nanoweapon, Trends Microbiol. 24 (2016) 51-62.

L.S. Ma, J.S. Lin, E.M. Lai, An IcmF family protein, ImpLM, is an integral inner membrane protein interacting with ImpKL, and its walker a motif is required for type VI secretion system-mediated Hcp secretion in Agrobacterium tumefaciens, J Bacteriol. 191 (2009) 43164329.

[10] M.S. Aschtgen, M. Gavioli, A. Dessen, R. Lloubès, E. Cascales, The SciZ protein anchors the enteroaggregative Escherichia coli type VI secretion system to the cell wall, Mol. Microbiol.

[11] E. Durand, V.S. Nguyen, A. Zoued, L. Logger, G. Péhau-Arnaudet, M.S. Aschtgen, S. Spinelli, 75 (2010) 886-899.

[12] Y.R. Brunet,A. Zoued, F.Boyer,B. Douzi, E.Cascales, The type VI secretion TssEFGK-VgrG phage-like baseplate is recruited to the TssJLM membrane complex via multiple contacts and serves as assembly platform for tail tube/sheath polymerization, PLoS Genet. 15 (3) (2015) e1005545.

[13] A. Zoued, C.J. Cassaro, E. Durand, B. Douzi, A.P. España, C. Cambillau, L. Journet, E. Cascales, Structure-function analysis of the TssL cytoplasmic domain reveals a new interaction between the Type VI secretion baseplate and membrane complexes, J Mol Biol. 428 (2016) 4413-4423. 
1 [14] L. Logger, M.S. Aschtgen, M. Guérin, E. Cascales, E. Durand, Molecular dissection of the interface between the Type VI secretion TssM cytoplasmic domain and the TssG baseplate component, J Mol Biol. 428 (2016) 4424-4437.

[15] V.S. Nguyen, L. Logger, S. Spinelli, P. Legrand, T.T. Huyen Pham, T.T. Nhung Trinh, Y. Cherrak, A. Zoued, A. Desmyter, E. Durand, A. Roussel, C. Kellenberger, E. Cascales, C. Cambillau, Type VI secretion TssK baseplate protein exhibits structural similarity with phage receptor-binding proteins and evolved to bind the membrane complex, Nat Microbiol. 2 (2017) 17103.

[16] A. Zoued, Y.R. Brunet, E. Durand, M.S. Aschtgen, L. Logger, B. Douzi, L. Journet, C. Cambillau, E. Cascales, Architecture and assembly of the type VI secretion system, Biochim. Biophys. Acta 1843 (2014) 1664-1673.

[17] B.T. Ho, T.G. Dong, J.J. Mekalanos, A view to a kill: the bacterial Type VI secretion system, Cell Host Microbe 15 (2014) 9-21.

[18] P.F. Sarris, E.D. Ladoukakis, N.J. Panopoulos, E.V. Scoulica, A phage tail-derived element with wide distribution among both prokaryotic domains: a comparative genomic and phylogenetic study. Genome Biol Evol. 6 (2014) 1739-1747.

[19] M. Basler, Type VI secretion system: secretion by a contractile nanomachine, Philos. Trans. R. Soc. Lond. Ser. B Biol. Sci. 370 (2015) 1679.

[20] D. Böck, J.M. Medeiros, H.F. Tsao, T. Penz, G.L. Weiss, K. Aistleitner, M. Horn, M. Pilhofer, In situ architecture, function, and evolution of a contractile injection system, Science. 357 (2017) 713-717.

[21] E. Cascales, Microbiology: and Amoebophilus invented the machine gun!, Curr Biol. 27 (2017) 1170-1173.

[22] P.G. Leiman, M.M. Shneider, Contractile tail machines of bacteriophages, Adv. Exp. Med. Biol. 726 (2012) 93-114.

[23] G. Bönemann, A. Pietrosiuk, A. Mogk, Tubules and donuts: a type VI secretion story, Mol Microbiol. 76 (2010) 815-821. 
1 [24] J.B. Heymann, J.D. Bartho, D. Rybakova, H.P. Venugopal, D.C. Winkler, A. Sen, M.R. Hurst,

A.K. Mitra, Three-dimensional structure of the toxin-delivery particle antifeeding prophage of Serratia entomophila, J Biol Chem. 288 (2013) 25276-25284.

[25] Y. Michel-Briand, C. Baysse, The pyocins of Pseudomonas aeruginosa, Biochimie 84 (2002) 499-510.

[26] N.J. Shikuma, M. Pilhofer, G.L. Weiss, M.G. Hadfield, G.J. Jensen, D.K. Newman, Marine tubeworm metamorphosis induced by arrays of bacterial phage tail-like structures, Science 343 (2014) 529-533.

[27] T. Penz, M. Horn, S. Schmitz-Esser, The genome of the amoeba symbiont "Candidatus Amoebophilus asiaticus" encodes an afp-like prophage possibly used for protein secretion, Virulence 1 (2010) 541-545.

[28] M. Brackmann, S. Nazarov, J. Wang, M. Basler, Using force to punch holes: mechanics of contractile nanomachines, Trends Cell Biol. 27 (2017) 623-632.

[29] T. Jank, S. Eckerle, M. Steinemann, C. Trillhaase, M. Schimpl, S. Wiese, D.M. van Aalten, W. Driever, K. Aktories, Tyrosine glycosylation of Rho by Yersinia toxin impairs blastomere cell behaviour in zebrafish embryos, Nat Commun. 6 (2015) 7807.

[30] A. Hachani, T.E. Wood, A. Filloux, Type VI secretion and antihost effectors, Curr. Opin. Microbiol. 29 (2015) 81-93.

[31] C.R. Büttner, Y. Wu, K.L. Maxwell, A.R. Davidson, Baseplate assembly of phage Mu: Defining the conserved core components of contractile-tailed phages and related bacterial systems, Proc Natl Acad Sci U S A. 113 (2016) 10174-10179.

[32] F. Arisaka, M.L. Yap, S. Kanamaru, M.G. Rossmann, Molecular assembly and structure of the bacteriophage T4 tail, Biophys Rev. 8 (2016) 385-396.

[33] N.M. Taylor, N.S. Prokhorov, R.C. Guerrero-Ferreira, M.M. Shneider, C. Browning, K.N. Goldie, H. Stahlberg, P.G. Leiman, Structure of the T4 baseplate and its function in triggering sheath contraction, Nature 533 (2016) 346-352.

[34] N.R. Watts, D.H. Coombs, Structure of the bacteriophage T4 baseplate as determined by chemical cross-linking, J Virol. 64 (1990) 143-154. 
1 [35] S. Kanamaru, P.G. Leiman, V.A. Kostyuchenko, P.R. Chipman, V.V. Mesyanzhinov, F. Arisaka, M.G. Rossmann, Structure of the cell-puncturing device of bacteriophage T4, Nature 415 (2002) 553-557.

[36] F. Arisaka, S. Kanamaru, Protein interactions in the assembly of the tail of bacteriophage T4, Biophys Rev. 5 (2013) 79-84.

[37] S. Pukatzki, A.T. Ma, A.T. Revel, D. Sturtevant, J.J. Mekalanos, Type VI secretion system translocates a phage tail spike-like protein into target cells where it cross-links actin, Proc. Natl. Acad. Sci. USA. 104 (2007) 15508-15513.

[38] P.G. Leiman, M. Basler, U.A. Ramagopal, J.B. Bonanno, J.M. Sauder, S. Pukatzki, S.K. Burley, S.C. Almo, J.J. Mekalanos, Type VI secretion apparatus and phage tail associated protein complexes share a common evolutionary origin, Proc. Natl. Acad. Sci. U. S. A. 106 (2009) 4154-4159.

[39] P.G. Leiman, F. Arisaka, M.J. van Raaij, V.A. Kostyuchenko, A.A. Aksyuk, S. Kanamaru, M.G. Rossmann, Morphogenesis of the T4 tail and tail fibers, Virol J. 7 (2010) 355.

[40] E. Cascales, C. Cambillau, Structural biology of type VI secretion systems, Philos. Trans. R. Soc. Lond. Ser. B Biol. Sci. 367 (2012) 1102-1111.

[41] J.S. Lin, L.S. Ma, E.M. Lai, Systematic dissection of the agrobacterium type VI secretion system reveals machinery and secreted components for subcomplex formation, PLoS ONE. 8 (2013) e67647.

[42] A. Zoued, E. Durand, C. Bebeacua, Y.R. Brunet, B. Douzi, C. Cambillau, E. Cascales, L. Journet, TssK is a trimeric cytoplasmic protein interacting with components of both phagelike and membrane anchoring complexes of the Type VI secretion system, J. Biol. Chem. 288 (2013) 27031-27041.

[43] M. Spínola-Amilibia, I. Davó-Siguero, F.M. Ruiz, E. Santillana, F.J. Medrano, A. Romero, The structure of VgrG1 from Pseudomonas aeruginosa, the needle tip of the bacterial type VI secretion system, Acta Crystallogr D Struct Biol. 72 (2016) 22-33.

[44] D. Veesler, C. Cambillau, A common evolutionary origin for tailed-bacteriophage functional modules and bacterial machineries, Microbiol Mol Biol Rev. 75 (2011) 423-433. 
[45] K. Uchida, P.G. Leiman, F. Arisaka, S. Kanamaru, Structure and properties of the C-terminal $\beta$-helical domain of VgrG protein from Escherichia coli O157, J Biochem. 155 (2014) 173182.

[46] D. Unterweger, B. Kostiuk, S. Pukatzki, Adaptor proteins of Type VI secretion system effectors, Trends Microbiol. 25 (2017) 8-10.

[47] Y.R. Brunet, J. Hénin, H. Celia, E. Cascales, Type VI secretion and bacteriophage tail tubes share a common assembly pathway, EMBO Rep. 15 (2014) 315-321.

[48] L.G. Pell, V. Kanelis, L.W. Donaldson, P.L. Howell, A.R. Davidson, The phage lambda major tail protein structure reveals a common evolution for long-tailed phages and the type VI bacterial secretion system, Proc Natl Acad Sci U S A. 106 (2009) 4160-4165.

[49] J.D. Mougous, M.E. Cuff, S. Raunser, A. Shen, M. Zhou, C.A. Gifford, A.L. Goodman, G. Joachimiak, C.L. Ordoñez, S. Lory, T. Walz, A. Joachimiak, J.J. Mekalanos, A virulence locus of Pseudomonas aeruginosa encodes a protein secretion apparatus. Science. 312 (2006) $1526-1530$.

[50] M. Kudryashev, R.Y. Wang, M. Brackmann, S. Scherer, T. Maier, D. Baker, F. DiMaio, H. Stahlberg, E.H. Egelman, M. Basler, Structure of the type VI secretion system contractile sheath, Cell 160 (2015) 952-962.

[51] A. Vettiger, J. Winter, L. Lin, M. Basler, The type VI secretion system sheath assembles at the end distal from the membrane anchor, Nat Commun. 8 (2017) 16088.

[52] J. Wang, M. Brackmann, D. Castano-Diez, M. Kudryashev, K.N. Goldie, T. Maier, H. Stahlberg, M. Basler, Cryo-EM structure of the extended type VI secretion system sheath-tube complex, Nat. Microbiol. 2 (2017) 1507-1512.

[53] A. Zoued, E. Durand, Y.R. Brunet, S. Spinelli, B. Douzi, M. Guzzo, N. Flaugnatti, P. Legrand, L. Journet, R. Fronzes, T. Mignot, C. Cambillau, E. Cascales, Priming and polymerization of a bacterial contractile tail structure, Nature 531 (2016) 59-63.

[54] A. Zoued, E. Durand, Y.G. Santin, L. Journet, A. Roussel, C. Cambillau, E. Cascales, TssA: The cap protein of the Type VI secretion system tail, Bioessays. 39 (2017) 262.

[55] N. Flaugnatti, T.T. Le, S. Canaan, M.S. Aschtgen, V.S. Nguyen, S. Blangy, C. Kellenberger, A. Roussel, C. Cambillau, E. Cascales, L. Journet, A phospholipase A1 antibacterial Type VI 
secretion effector interacts directly with the C-terminal domain of the VgrG spike protein for delivery, Mol Microbiol. 99 (2016) 1099-1118.

[56] A.J. Gerc, A. Diepold, K. Trunk, M. Porter, C. Rickman, J.P. Armitage, N.R. Stanley-Wall, S.J. Coulthurst, Visualization of the Serratia Type VI secretion system reveals unprovoked attacks and dynamic assembly, Cell Rep. 12 (2015) 2131-2142.

[57] A. Vettiger, M. Basler, Type VI secretion system substrates are transferred and reused among sister cells, Cell. 167 (2016) 99-110.

[58] E.R. Ballister, A.H. Lai, R.N. Zuckermann, Y. Cheng, J.D. Mougous, In vitro self-assembly of tailorable nanotubes from a simple protein building block, Proc. Natl. Acad. Sci. USA. 105 (2008) 3733-3738.

[59] B. Douzi, S. Spinelli, S. Blangy, A. Roussel, E. Durand, Y.R. Brunet, E. Cascales, C. Cambillau, Crystal structure and self-interaction of the Type VI Secretion tail-tube orotein from Enteroaggregative Escherichia coli, PLoS ONE 9 (2014) e86918.

[60] G. Karimova, J. Pidoux, A. Ullmann, D. Ladant, A bacterial two-hybrid system based on a reconstituted signal transduction pathway, Proc. Natl. Acad. Sci. USA. 95 (1998) 5752-5756.

[61] Y.R. Brunet, C.S. Bernard, M. Gavioli, R. Lloubes, E. Cascales, An epigenetic switch involving overlapping fur and DNA methylation optimizes expression of a type VI secretion gene cluster, PLoS Genetics 7 (2011) e1002205.

[62] M.S. Aschtgen, C.S. Bernard, S. De Bentzmann, R. Lloubès, E. Cascales, SciN is an outer membrane lipoprotein required for type VI secretion in enteroaggregative Escherichia coli, J. Bacteriol. 190 (2008) 7523-7531.

[63] F. van den Ent, J. Lowe, RF cloning: a restriction-free method for inserting target genes into plasmids, J. Biochem. Biophys. Meth. 67 (2006) 67-74.

[64] Y.R. Brunet, L. Espinosa, S. Harchouni, T. Mignot, E. Cascales, Imaging type VI secretionmediated bacterial killing, Cell Rep. 3 (2013) 36-41.

[65] G.C.P. van Zundert, J.P. Rodrigues, M. Trellet, C. Schmitz, P.L. Kastritis, E. Karaca, A.S.J. Melquiond, M. van Dijk, S.J. de Vries, A.M.J. Bonvin, The HADDOCK2.2 web server: userfriendly integrative modeling of biomolecular complexes, J Mol Biol. 428 (2016) 720-725. 


\section{Legend to Figures}

4 Figure 1. Hcp1 interacts with VgrG1. (A) Bacterial two-hybrid analysis. BTH101 reporter

5 cells producing the indicated proteins fused to the T18 and T25 domain of the Bordetella

6 adenylate cyclase were spotted on X-Gal-IPTG reporter LB agar plates. The blue color of the

7 colony reports interaction between the two partners. Controls include T18 and T25 fusions to

8 Pal and TolB, two proteins that interact but unrelated to the T6SS. (B and C) Co-

9 immunoprecipitation assay. Soluble lysates of E. coli cells producing the indicated proteins

$10\left(\right.$ VgrG1 $_{\mathrm{V}}$, VSV-G-tagged VgrG1; Hcp1 $\mathrm{F}$, FLAG-tagged Hcp1) were subjected to

11 immunoprecipitation with anti-FLAG (B) or anti-VSV-G (C) coupled beads. The total lysates

12 (T) and immunoprecipitated (IP) material were separated by $12.5 \%$ acrylamide SDS-PAGE

13 and immunodetected with anti-VSV-G (upper panel) and anti-FLAG (lower panel)

14 monoclonal antibodies. The position of $\mathrm{VgrG}_{\mathrm{V}}$ and $\mathrm{Hcp} 1_{\mathrm{F}}$ are indicated on the right.

15 Molecular weight markers (in $\mathrm{kDa}$ ) are indicated on the left.

Figure 2. The gp27-like base of VgrG1 is sufficient to mediate interaction with Hcp1. (A)

18 Schematic representation of the EAEC VgrG1 architecture. The different domains are

19 represented with a color code (blue, gp27; red, OB-fold; green, gp5-C; grey, coiled-coil, DUF2345 and TTR). The boundaries (in amino-acid) are indicated. The EAEC VgrG1 core domain has been modelled based on the crystal structure of the P. aeruginosa VgrG1 (PDB: 4MTK). (B) Schematic representations of VgrG1 and the deletion constructs used in this study. The color code is the same as in panel (A). (C) Co-immunoprecipitation assay. Soluble

24 lysates of E. coli cells producing the indicated VSV-G-tagged VgrG1 deletion variants and 
1 beads. The total lysates (T) and immunoprecipitated (IP) material were separated by $12.5 \%$

2 acrylamide SDS-PAGE and immunodetected with anti-VSV-G (upper panel) and anti-FLAG

3 (lower panel) monoclonal antibodies. The position of $\mathrm{Hcp} 1_{\mathrm{F}}$ is indicated on the right whereas

4 positions of the VgrG1 variants are indicated by asterisks. Molecular weight markers (in kDa)

5 are indicated on left. (D) Microscale thermophoresis. Left upper panel: Coomassie blue

6 staining of the VgrG1- $\Delta \mathrm{gp} 5$ and Hcp1 purified proteins subjected to SDS-PAGE. Molecular

7 weight markers are indicated on left. Right upper panel: Thermophoretic time trace

8 recordings of the unlabeled Hcp1 titration (from $0.3 \mathrm{nM}$ to $100 \mu \mathrm{M}$ ) to a constant amount of

9 fluorescently labeled VgrG1- $\Delta$ gp5. The measured changes in the MST response $(\Delta \mathrm{F})$ were

10 plotted against the Hcp1 concentration to estimate the binding constant $\left(\mathrm{K}_{D}=2.8 \pm 0.1 \mu \mathrm{M}\right)$

11 (lower panel).

Figure 3. The gp27-like base of VgrG1 is sufficient to promote Hcp1 tube formation,

sheath dynamics and Hcp1 release. (A) Hcp1 tube formation. Cytoplasmic extracts from $\Delta \operatorname{vgrG1}$ cells (left panel), or $\Delta \operatorname{vgrG1}$ cells producing $\operatorname{VgrG1}\left(\operatorname{vgr} G 1^{+}\right.$, middle panel) or VgrG1- $\triangle \mathrm{OB}(\triangle \mathrm{OB}$, right panel) and FLAG-tagged Hcp1 (-) or the indicated Hcp1 variants reporting head-to-head (H-to-H, G48C), tail-to-tail (T-to-T, Q24C/A95C) or head-to-tail (Hto-T, G96C/S158C) assemblies after in vivo oxidative treatment were analyzed by $12.5 \%$ acrylamide SDS-PAGE and immunodetected with the anti-FLAG monoclonal antibody. Positions of the Hcp1 monomer $\left(\operatorname{Hcp} 1_{\mathrm{F}}\right)$ and oligomers $\left(\operatorname{Hcp} 1_{\mathrm{F}}{ }^{*}\right)$ are indicated on the right. Molecular weight markers (in $\mathrm{kDa}$ ) are indicated on the left. (B) Sheath dynamics. Time-lapse recordings of $\Delta v g r G 1$ cells carrying the empty pBAD33 vector ( $\operatorname{vgr} G 1)$, the pBAD33 vector producing VgrG1 $\left(\operatorname{vgr} G 1^{+}\right)$or $\operatorname{VgrG} 1-\Delta \mathrm{OB}(\operatorname{vgr} G 1-\Delta \mathrm{OB})$ and producing the TssB-mCherry fusion were monitored for $150 \mathrm{sec}$. Sheath extension and contraction events are pointed using open and close arrowheads respectively. Scale bar is $1 \mu \mathrm{M}$. Statistical analyses of sheath 
1 dynamics are shown in Fig. S3. (C) Hcp1 release assay. FLAG-tagged Hcp1 (Hcp1 $\left.1_{\mathrm{F}}\right)$ release

2 was assessed by separating total cell (C) and cell-free culture supernatant (SN) fractions from

$35 \times 10^{8} \Delta v g r G 1$ cells carrying the empty pBAD33 vector $(\operatorname{vgr} G 1)$, the pBAD33 vector 4 producing VgrG1 $\left(\operatorname{vgr} G 1^{+}\right)$or VgrG1- $\triangle \mathrm{OB}(\operatorname{vgr} G 1-\Delta \mathrm{OB})$. Proteins were separated by $12.5 \%$ 5 acrylamide SDS-PAGE and periplasmic TolB and Hcp $1_{\mathrm{F}}$ were immunodetected using anti6 TolB (upper panel) and anti-FLAG (lower panel) antibodies. Molecular weight markers (in $7 \mathrm{kDa}$ ) are indicated on the left.

Figure 4. The head and tail sides of the Hcp1 hexamer engage contacts with VgrG1 and

TssA respectively. (A) Bacterial two-hybrid analysis. BTH101 reporter cells producing the indicated proteins and variants fused to the T18 and T25 domain of the Bordetella adenylate cyclase were spotted on X-Gal-IPTG reporter LB agar plates. The blue color of the colony reports interaction between the two partners. Controls include T18 and T25 fusions to Pal and TolB, two proteins that interact but unrelated to the T6SS. (B) Co-immunoprecipitation assay. Soluble lysates of $E$. coli cells producing the indicated proteins or protein variants were subjected to immunoprecipitation with anti-FLAG-coupled beads. The total lysates (T) and immunoprecipitated (IP) material were separated by $12.5 \%$ acrylamide SDS-PAGE and immunodetected with anti-VSV-G (upper panel) and anti-FLAG (lower panel) monoclonal antibodies. The position of $\mathrm{VgrG}_{\mathrm{V}}$ and $\mathrm{Hcp} 1_{\mathrm{F}}$ are indicated on the right. Molecular weight markers (in $\mathrm{kDa}$ ) are indicated on the left. (C) VgrG1-Hcp1 interface. The structural model of the EAEC VgrG1 protein (dark grey) and the structure of the EAEC Hcp1 hexameric ring (light grey; PDB: 4HKH) are shown, highlighting the locations of the Hcp1 tryptophan substitutions (red, N93W variant; green, S158W variant) and of three VgrG1 loops (red, Ser115 to Asn-120; green, Pro-219 to Ser-229; cyan, Ser-360 to Lys-362) at the base of the VgrG gp27-like hub domain. The faces of Hcp1 engaged in the interactions with VgrG1 and TssA 
1 are indicated with arrows. (D) Bacterial two-hybrid analysis. BTH101 reporter cells

2 producing the indicated proteins and variants fused to the T18 and T25 domain of the

3 Bordetella adenylate cyclase were spotted on X-Gal-IPTG reporter LB agar plates. The blue

4 color of the colony reports interaction between the two partners. Controls include T18 and

5 T25 fusions to Pal and TolB, two proteins that interact but unrelated to the T6SS. (E) Hcp1

6 tube formation. Cytoplasmic extracts from $\Delta v g r G 1$ cells producing the $\mathrm{VgrG} 1_{\text {loop }}$ variant and

7 FLAG-tagged Hcp1 (-) or the indicated Hcp1 variants reporting head-to-head (H-to-H, G48C),

8 tail-to-tail (T-to-T, Q24C/A95C) or head-to-tail (H-to-T, G96C/S158C) assemblies after in

9 vivo oxidative treatment were analyzed by $12.5 \%$-acrylamide SDS-PAGE and

10 immunodetected with the anti-FLAG monoclonal antibody. Positions of the Hcp1 monomer

$11\left(\mathrm{Hcp} 1_{\mathrm{F}}\right)$ and oligomers $\left(\mathrm{Hcp} 1_{\mathrm{F}}{ }^{*}\right)$ are indicated on the right. Molecular weight markers (in $\left.\mathrm{kDa}\right)$

12 are indicated on the left. (F) E. coli $\mathrm{K}-12$ recipient cells (W3110 $g f p^{+}, \mathrm{kan}^{\mathrm{R}}$ ) were mixed with

13 the indicated attacker cells (WT, EAEC 17-2; $\Delta v g r G 1, \Delta v g r G 1$ cells carrying the pBAD33

14 empty vector; $\operatorname{vgr} G 1^{+}, \Delta \operatorname{vgr} G 1$ cells producing $\mathrm{VgrG} 1 ; \operatorname{vgr} G 1_{\text {loop }}, \Delta \operatorname{vgr} G 1$ cells producing the

$15 \mathrm{VgrG}_{\text {loop }}$ variant, spotted onto Sci-1 inducing medium (SIM) agar plates and incubated for 4

16 hours at $37^{\circ} \mathrm{C}$. The image of a representative bacterial spot is shown below the graph

17 reporting the number of surviving E. coli prey cells (counted on selective kanamycin medium;

18 in $\log 10$ of colony-forming units (cfu)). The open circles indicate values from three

19 independent assays, and the average is indicated by the bar.

21 Figure 5. Disulfide bond formation between VgrG1 and Hcp1. Cytoplasmic extracts from 22 cells producing the indicated VSV-G-tagged VgrG1 and FLAG-tagged Hcp1 cysteine variants 23 after in vivo oxidative treatment were subjected to $10 \%$-acrylamide SDS-PAGE and 24 immunodetected with the anti-VSV-G (upper panels in panel A, B and C; "V" panels in panel 25 D) and anti-FLAG (lower panels in panel A, B and C; "F" panels in panel D) monoclonal 
1 antibodies. In panel $\mathrm{C}$, samples were treated $(+)$ or not (-) with $\beta$-mercaptoethanol. In panel D,

2 each lane was cut vertically in two halves after protein transfer on nitrocellulose membrane 3 (dotted lines) and each half was immuno-detected with the anti-VSV-G (V) or anti-FLAG (F)

4 antibody. Positions of the Hcp1 (Hcp1 $\left.1_{\mathrm{F}}\right)$ and VgrG1 (VgrG1 $\left.\mathrm{V}\right)$ monomers, and of VgrG15 Hcp1 complexes (* and **) are indicated on the right. Molecular weight markers (in $\mathrm{kDa})$ are 6 indicated on the left.

8 Figure 6. Model of the EAEC VgrG1-Hcp1 complex. The modelled EAEC VgrG1 trimer 9 (same color code as Fig. 2A) is shown, as well as the Hcp1 hexameric ring (grey, PDB: $104 \mathrm{HKH})$. VgrG1 and Hcp1 are docked based on the position of cysteine pairs that engage in 11 disulfide bond formation (Hcp1 N46C and G48C, yellow; VgrG1 R361C and K362C, purple). 12 A magnification is shown on right.

14 Figure 7. Model for the early stages of T6SS tail biogenesis. The assembly of the T6SS tail 15 tube/sheath starts after docking of the baseplate (BC) onto the membrane complex (MC) (1) $16[11,12]$. The first ring of Hcp hexamer is recruited and docks to the VgrG base (2). Then, 17 Hcp hexameric rings and TssBC strands are recruited and the tail tube/sheath extends from 18 the distal end (3) $[51,53]$. The VgrG protein is shown with the same color code as Fig. 2A 19 and Fig. 6. The tail tube/sheath coordinator TssA is shown in red. OM, outer membrane; PG, 20 peptidoglycan; IM, inner membrane. 


\section{Legend to Supplementary Figures}

2

Figure S1. Microscale thermophoresis affinity measurements between cognate and noncognate VgrG and Hcp proteins. Thermophoretic time trace recordings of the unlabeled EAEC Hcp1 or Hcp2, or V. cholerae Hcp $\left(\operatorname{Hcp}_{v_{c}}\right.$ ) titration (from $0.9 \mathrm{nM}$ to $600 \mu \mathrm{M}$ ) to a

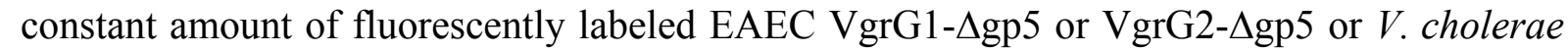
$\operatorname{VgrG}_{\mathrm{Vc}}-\Delta g p 5$ (left panels). The measured changes in the MST response $(\Delta \mathrm{F})$ were plotted against the Hcp concentration to calculate the binding constant (right panels). The VgrG2$\Delta \mathrm{gp} 5-\mathrm{Hcp} 2\left(\right.$ panel $\left.\mathrm{A}, \mathrm{K}_{D}=9.4 \pm 0.7 \mu \mathrm{M}\right), \operatorname{VgrG}_{\mathrm{Vc}_{\mathrm{c}}}-\Delta \mathrm{gp} 5-\mathrm{Hcp}_{\mathrm{Vc}}\left(\right.$ panel B, $\mathrm{K}_{D}=4.1 \pm 0.4$ $\mu \mathrm{M}$ ), VgrG1- $\Delta \mathrm{gp} 5-\mathrm{Hcp} 2\left(\right.$ panel $\mathrm{C}, \mathrm{K}_{D}=35.4 \pm 6 \mu \mathrm{M}$ ) and VgrG2- $\Delta$ gp5 - Hcp1 (panel D, $\mathrm{K}_{D}=22.4 \pm 1.9 \mu \mathrm{M}$ ) recordings are shown.

Figure S2. VgrG2 and Hcp2 do not compensate the absence of VgrG1 and Hcp1 for Sci1 T6SS-mediated inter-bacterial activity. E. coli $\mathrm{K}-12$ recipient cells (W3110 $g f p^{+}, \mathrm{kan}^{\mathrm{R}}$ ) were mixed with the indicated attacker cells: WT, EAEC 17-2; $\Delta v g r G 1, \Delta v g r G 1$ cells carrying the pBAD33 empty vector; $\operatorname{vgr} G 1^{+},{ } \operatorname{vgr} G 1$ cells producing $\operatorname{VgrG} 1 ; \operatorname{vgr} G 2^{+}$, $\Delta v g r G 1$ cells producing VgrG2 (panel A); WT, EAEC 17-2; $\Delta h c p 1, \Delta h c p 1$ cells carrying the pUC12 empty vector; $h c p 1^{+}, \Delta h c p 1$ cells producing Hcp1; $h c p 2^{+}, \Delta h c p 1$ cells producing Hcp2 (panel B). The mixtures were spotted onto Sci-1 inducing medium (SIM) agar plates and incubated for 4 hours at $37^{\circ} \mathrm{C}$. The image of a representative bacterial spot is shown below the graph reporting the number of surviving E. coli prey cells (counted on selective kanamycin medium; in $\log 10$ of colony-forming units (cfu)). The open circles indicate values from three independent assays, and the average is indicated by the bar. 
1 Figure S3. Statistical analyses of sheath dynamics. Distribution of the dynamic behavior of

2 T6SS sheaths, monitored by time-lapse fluorescence recordings of the indicated strain (WT,

3 EAEC 17-2; $\Delta v g r G 1, \Delta v g r G 1$ cells carrying the pBAD33 empty vector; $v g r G 1^{+}, \Delta v g r G 1$ cells

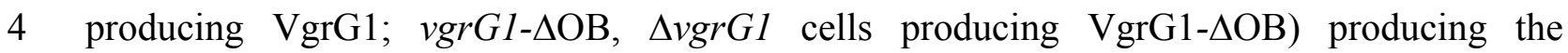
5 chromosomally-encoded $t s s B$-mCherry fusion. The bars represent the percentage of non6 dynamic tubular structures (blue) and sheaths that undergo cycles of extension-contraction 7 (green). The number of cells analysed $(n)$ is indicated on top. The asterisk $\left(^{*}\right)$ indicates that no 8 sheath is observable in this strain (diffuse fluorescence).

Figure S4. The gp27-like base of VgrG1 does not require VgrG2 to promote Hcp1 release. FLAG-tagged Hcp1 $\left(\mathrm{Hcp} 1_{\mathrm{F}}\right)$ release was assessed by separating total cell $(\mathrm{C})$ and cell-free culture supernatant (SN) fractions from $5 \times 10^{8} \Delta v g r G 1-\Delta v g r G 2$ cells carrying the empty pBAD33 vector (-), the pBAD33 vector producing $\operatorname{VgrG1}\left(\operatorname{vgr} G 1^{+}\right)$, VgrG1- $\Delta \mathrm{OB}$ ( $\operatorname{vg} G 1-\Delta \mathrm{OB})$ or $\operatorname{VgrG} 2\left(\operatorname{vgr} G 2^{+}\right)$. Proteins were separated by $12.5 \%$-acrylamide SDS-PAGE and periplasmic TolB and Hcp1 $1_{\mathrm{F}}$ were immunodetected using anti-TolB (upper panel) and anti-FLAG (lower panel) antibodies. Molecular weight markers (in kDa) are indicated on the left.

Figure S5. Hcp1 and VgrG1 cysteine variants are functional for T6SS-dependent interbacterial activity. E. coli $\mathrm{K}-12$ recipient cells (W3110 $g f p^{+}, \mathrm{kan}^{\mathrm{R}}$ ) were mixed with the indicated attacker cells (WT, EAEC 17-2; $\Delta h c p 1, \Delta h c p 1$ cells carrying the pUC12 empty vector; $h c p 1^{+}, \Delta h c p 1$ cells producing Hcp1; N46C, $\Delta h c p 1$ cells producing the Hcp1 N46C variant; $\Delta v g r G 1, \Delta v g r G 1$ cells carrying the pBAD33 empty vector; $\operatorname{vgr} G 1^{+}, \Delta v g r G 1$ cells producing VgrG1; R361C and K362C, $\Delta v g r G 1$ cells producing the VgrG1 R361C and K362C variants, respectively), spotted onto Sci-1 inducing medium (SIM) agar plates and incubated 
1 for 4 hours at $37^{\circ} \mathrm{C}$. The image of a representative bacterial spot is shown below the graph

2 reporting the number of surviving E. coli prey cells (counted on selective kanamycin medium;

3 in $\log 10$ of colony-forming units (cfu)). The open circles indicate values from three 4 independent assays, and the average is indicated by the bar. 


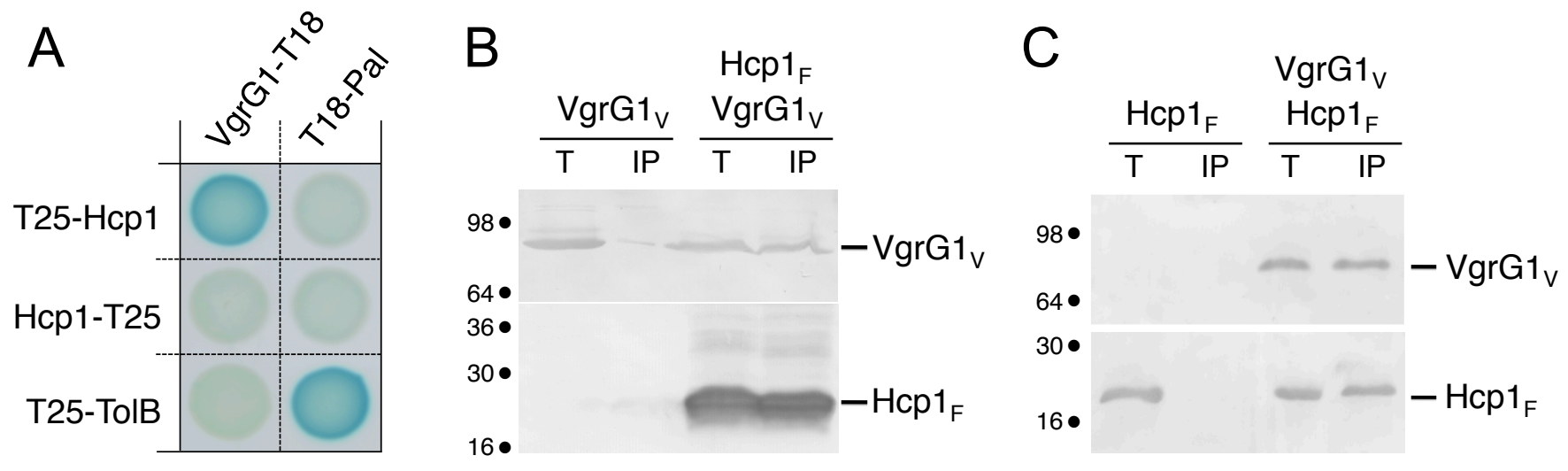


A

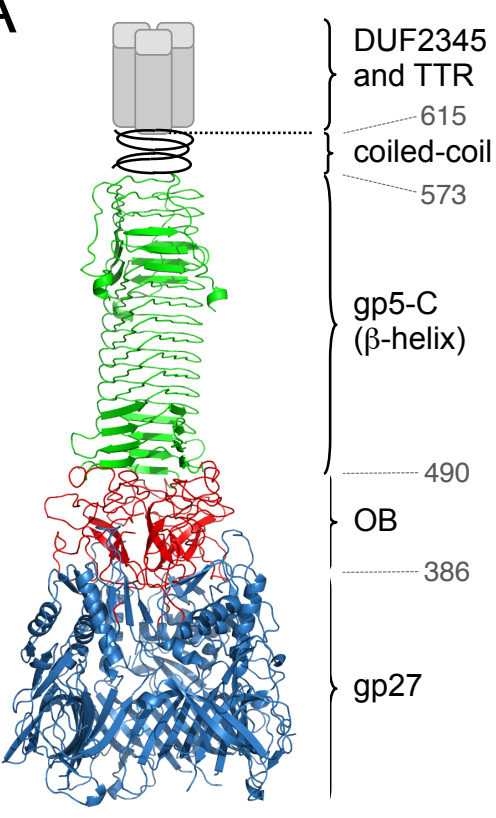

C

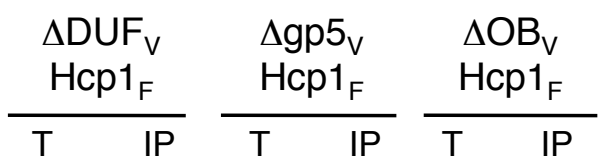

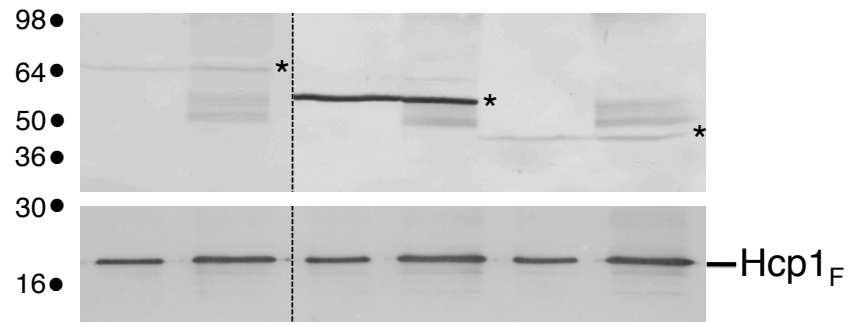

B

VgrG1

$\triangle \mathrm{DUF}$

$\Delta$ gp5

$\Delta \mathrm{OB}$
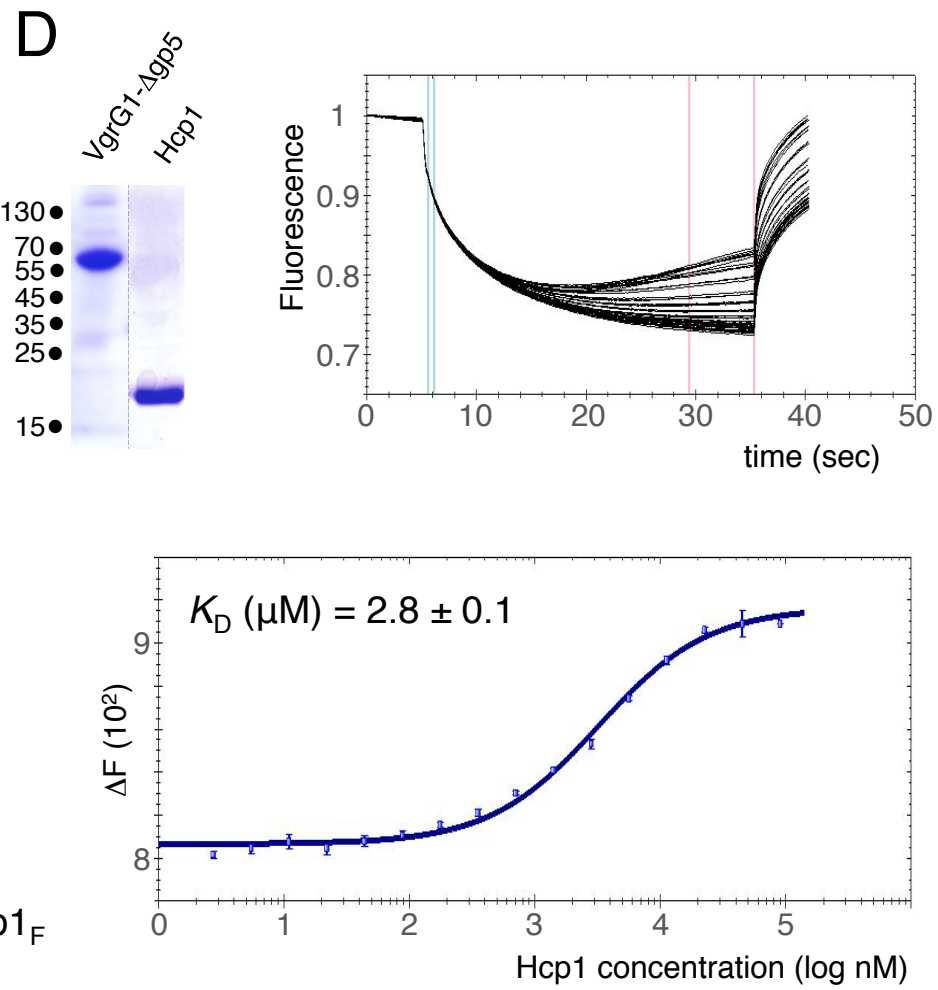
A

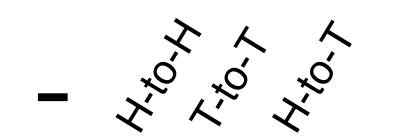

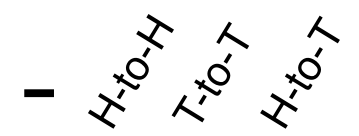<smiles>C[14CH]=[14CH]C</smiles>

250

$64 \bullet$

$50 \bullet$

$36 \bullet$

$30 \bullet$

$16 \bullet$

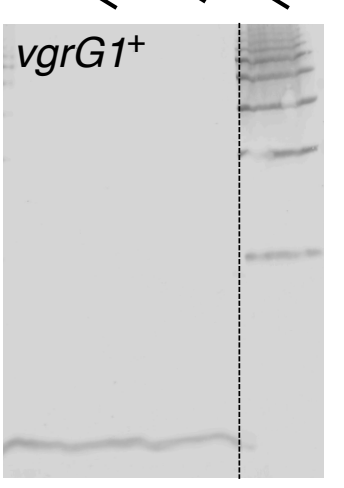

$\triangle \mathrm{OB}$

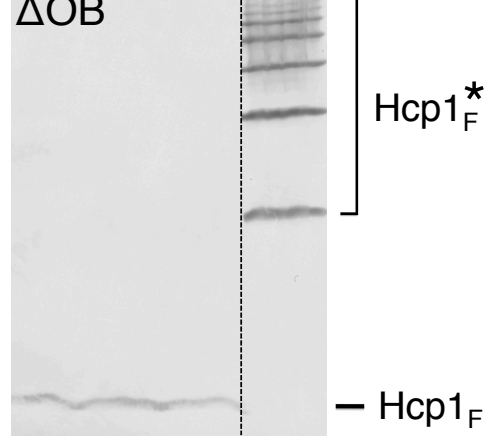

B

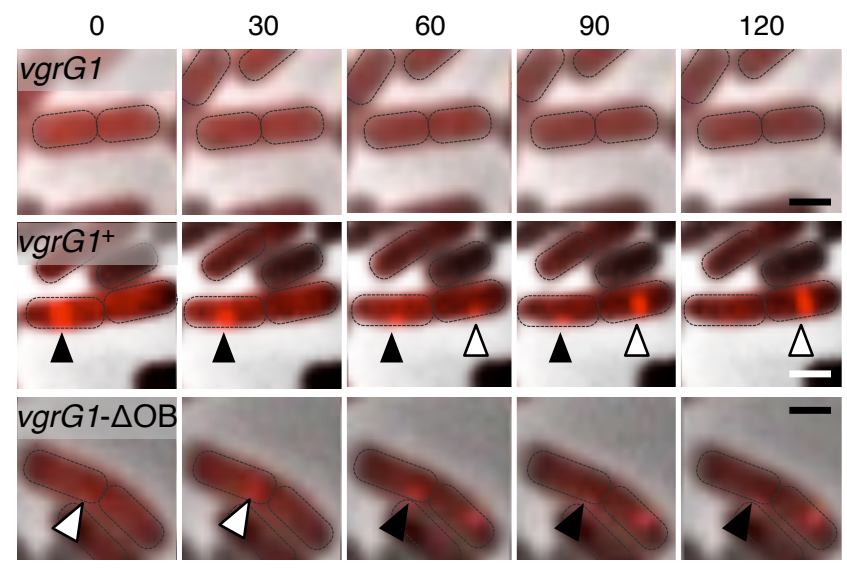

C

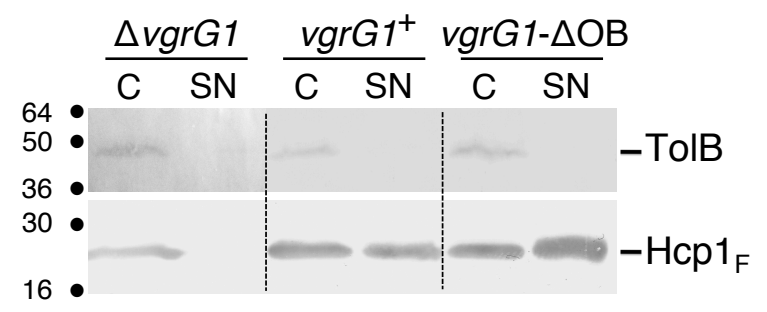

Figure 3 
A

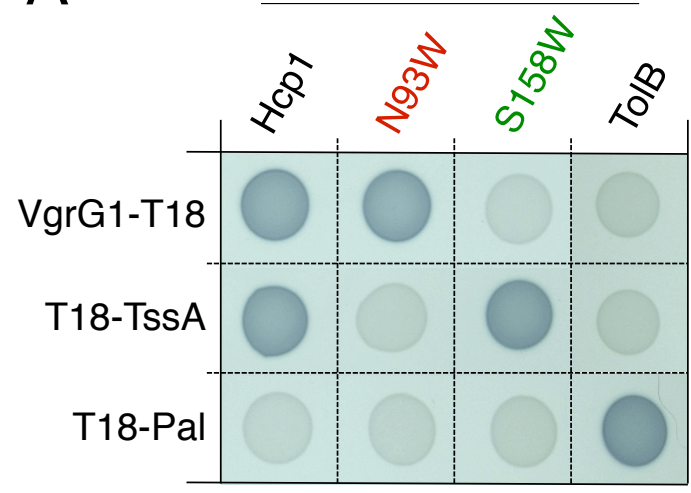

B $\quad V_{g r G} 1_{V} \quad V_{g r G 1} \quad \operatorname{VgrG} 1_{\text {loop }}$ $\frac{\mathrm{Hcp}_{\mathrm{F}}}{\mathrm{T} \quad \mathrm{IP}} \frac{\mathrm{Hcp} 1_{\mathrm{S} 158 \mathrm{~W}}}{\mathrm{~T} \quad \mathrm{IP}} \frac{\mathrm{Hcp}_{\mathrm{F}}}{\mathrm{T} \text { IP }}$

$98 \bullet$

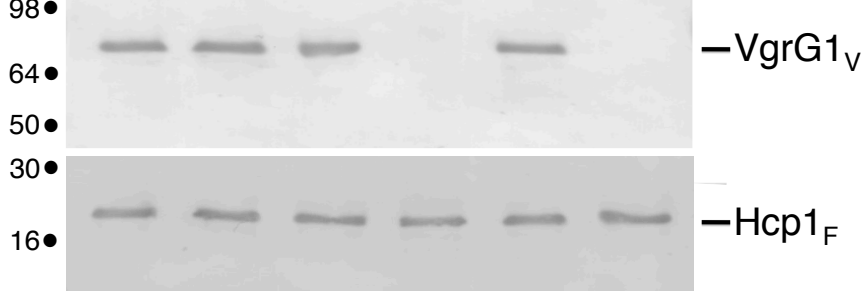

C

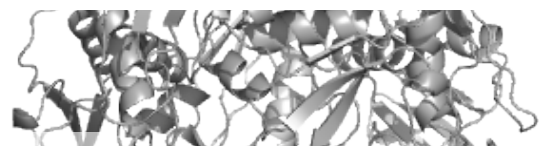

VgrG1
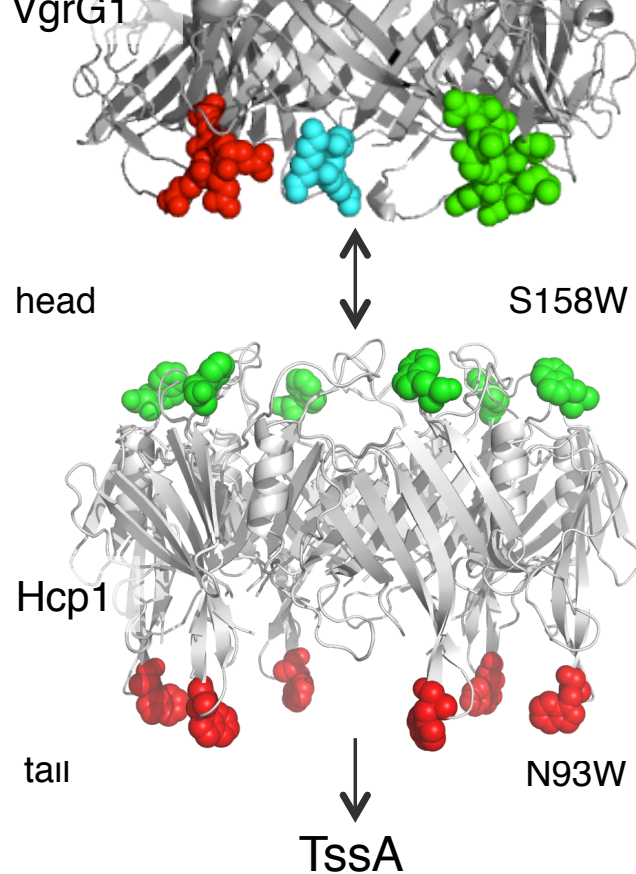

D

E

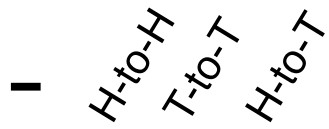

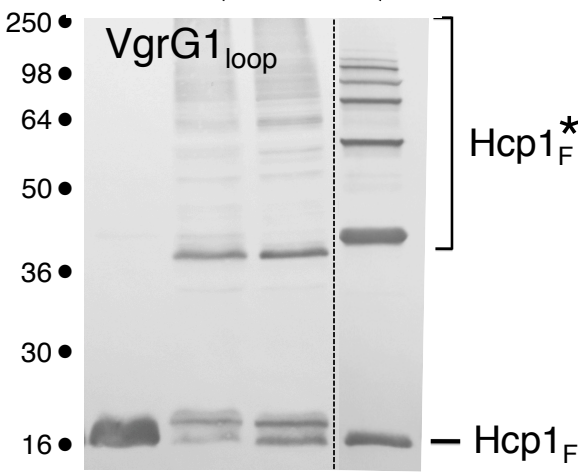

F

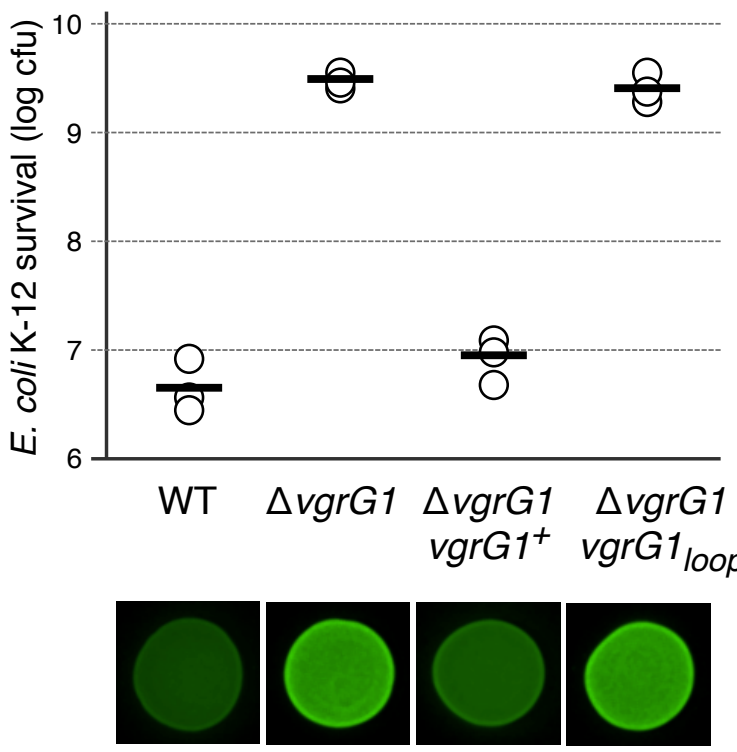




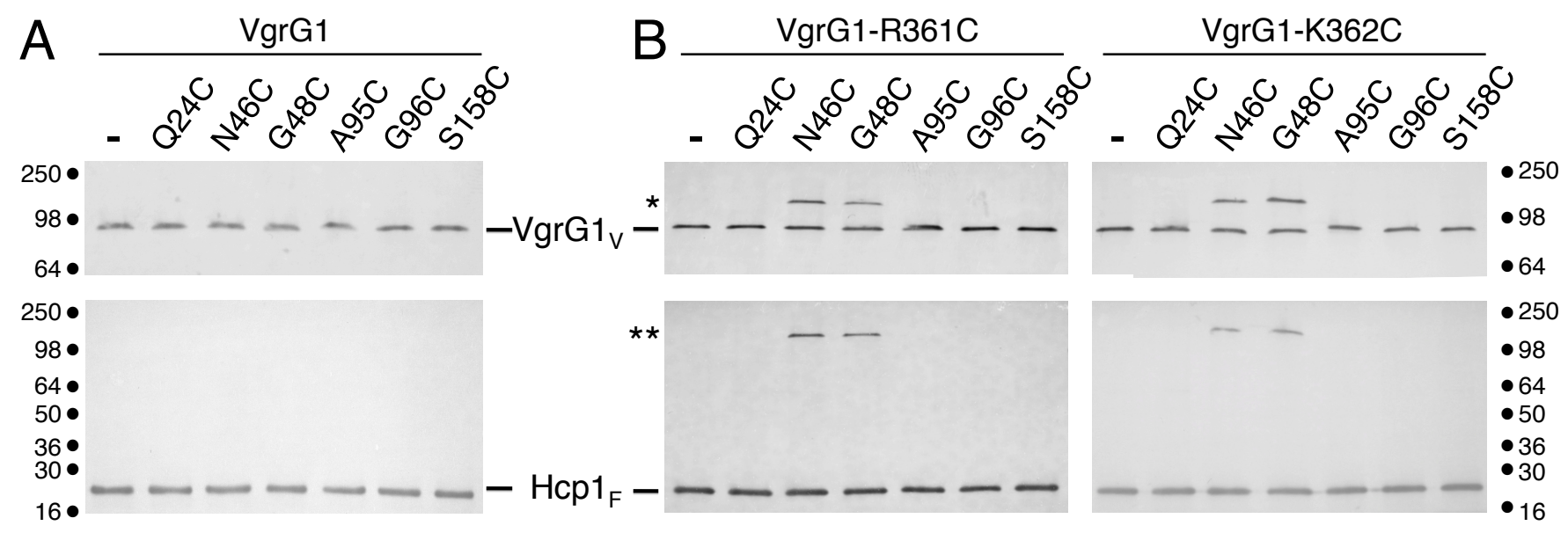

C $\frac{\text { VgrG1-R361C }}{\text { N46C G48C }} \frac{\text { VgrG1-K362C }}{\text { N46C G48C }}$

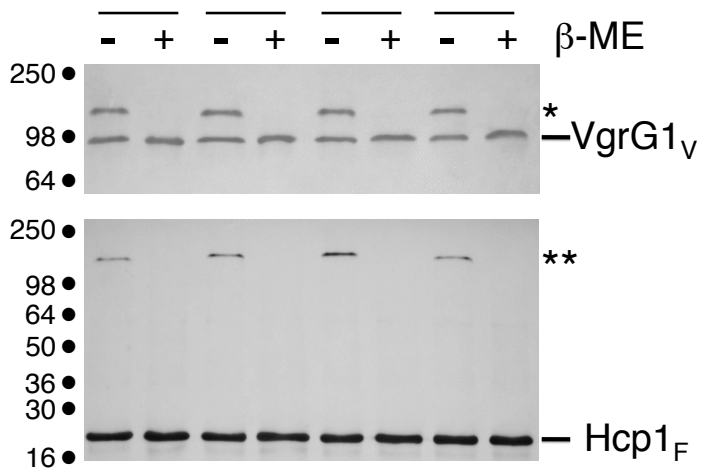

D $\frac{\text { VgrG1-R361C }}{\text { N46C G48C }} \frac{\text { VgrG1-K362C }}{\text { N46C G48C }}$

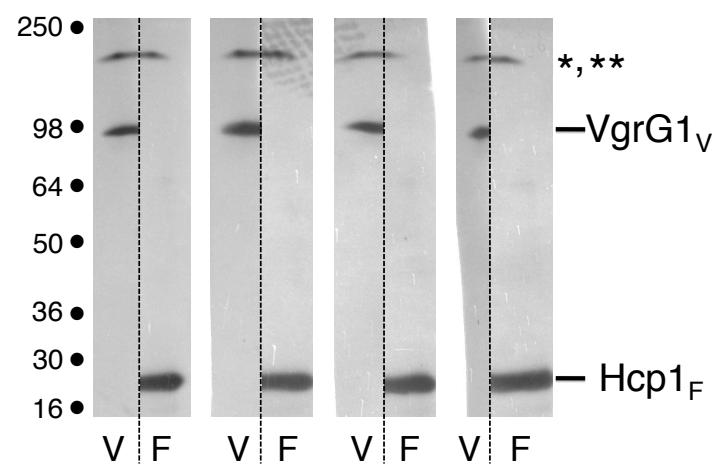



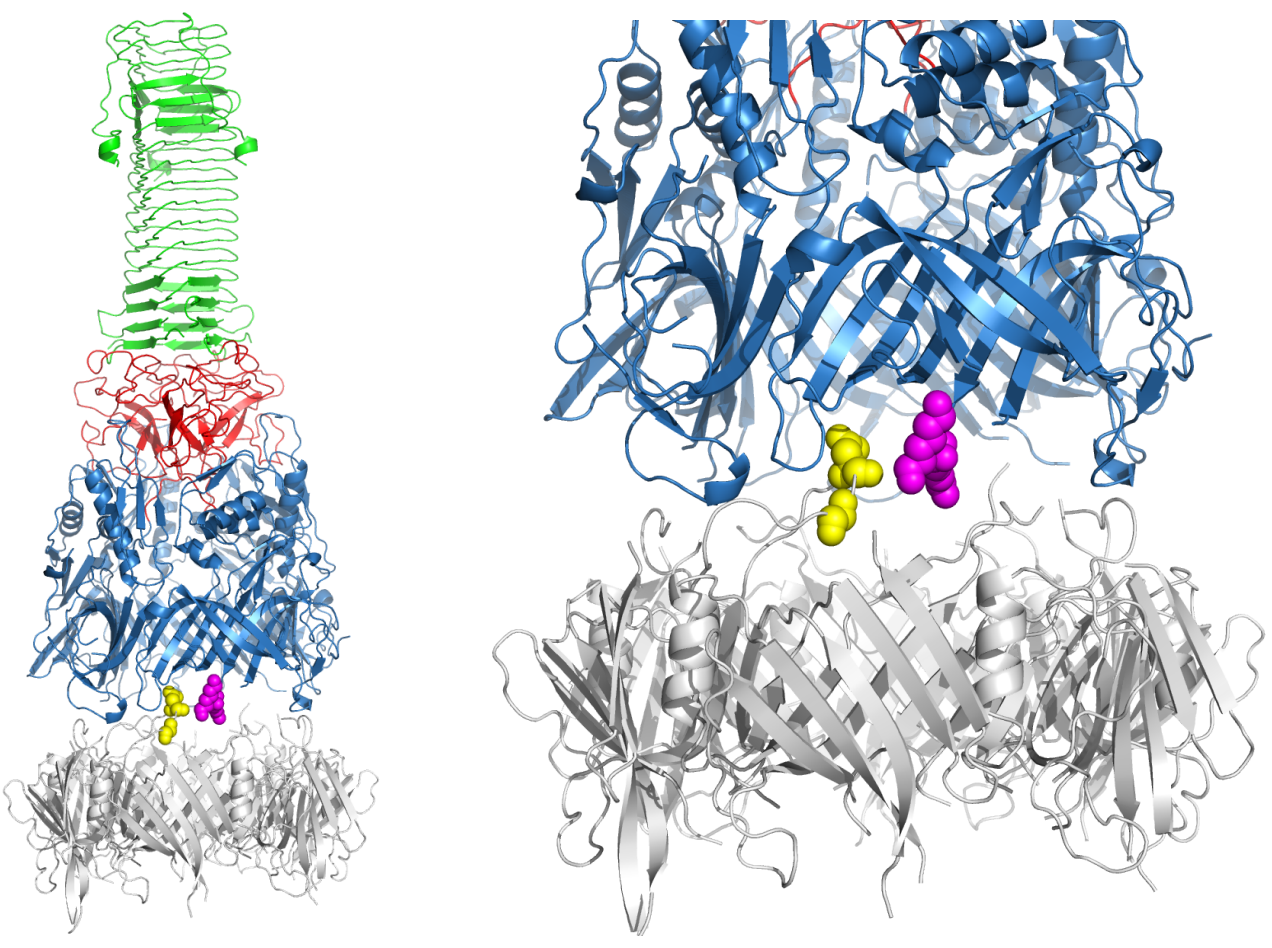

Figure 6 


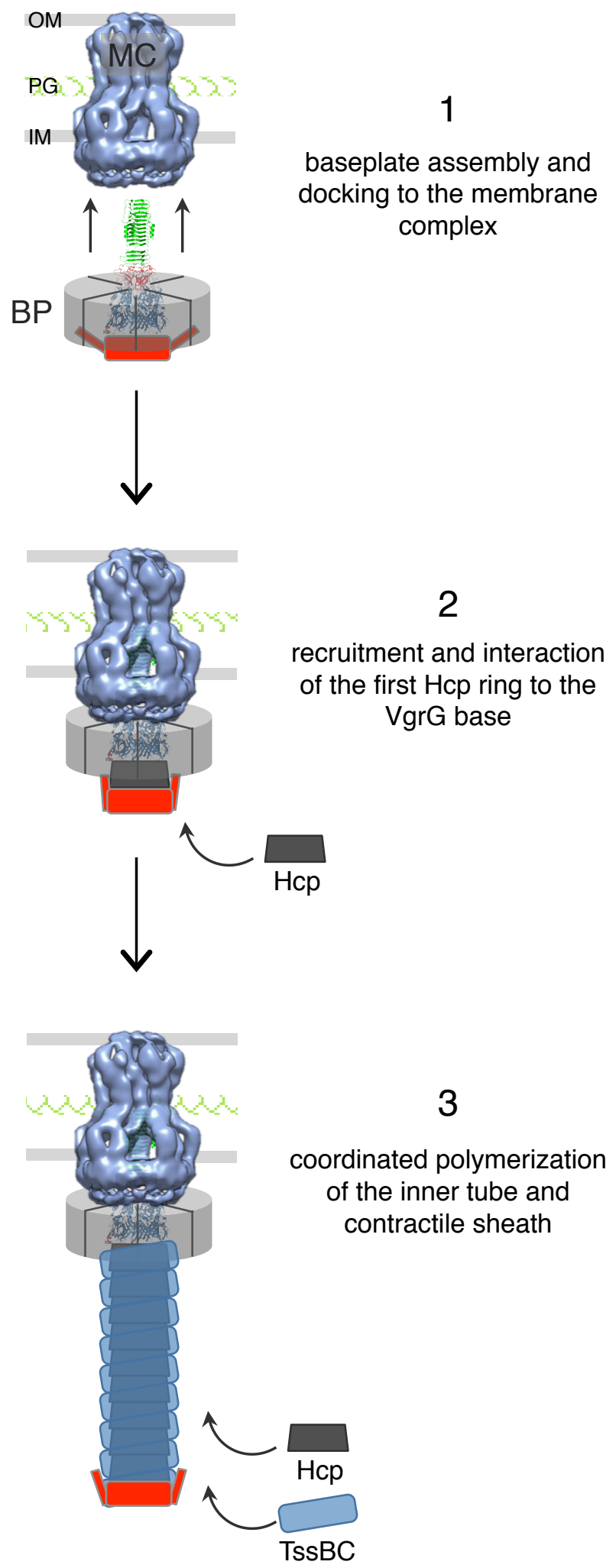


Table S1. Strains, plasmids and oligonucleotides used in this study.

\section{STRAINS}

$\begin{array}{lll}\text { Strains } & \text { Description } & \text { Source/Reference }\end{array}$

Enteroaggregative E. coli

$\begin{array}{lll}17-2 & \text { Wild-type enteroaggregative Escherichia coli } & \text { A. Darfeuille-Michaud } \\ \Delta v g r G & 17-2 \text { deleted of the } s c i-1 \text { vgrG gene } & \text { Brunet } \text { et al., } 2014 \\ \Delta h c p & 17-2 \text { deleted of the } s c i-1 \text { hcp gene } & \text { Zhang } \text { et al., 2013 } \\ \Delta v g r G \Delta h c p & \Delta v g r G \text { deleted of the } s c i-1 \text { hcp gene } & \text { This study } \\ \Delta v g r G t s s-m C h & \Delta v g r G \text { with mCherry inserted at the 3' of } t s s B & \text { This study }\end{array}$

E. coli $\mathrm{K}-12$

\begin{tabular}{|c|c|c|}
\hline DH5 $\alpha$ & 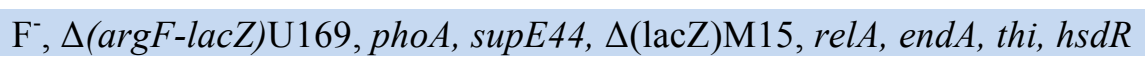 & New England Biolabs \\
\hline BTH101 & $\mathrm{F}^{-}$, cya-99, araD139, galE15, galK16, rpsL, hsdR, mcrA1, mcrB1 & Karimova et al., 1998 \\
\hline BL21(DE3)pLys & $\mathrm{F}^{-}$ompT gal dcm lon $h s d S_{\mathrm{B}}\left(\mathrm{r}_{\mathrm{B}}^{-} \mathrm{m}_{\mathrm{B}}^{-}\right) \lambda(\mathrm{DE} 3) \mathrm{pLysS}\left(\mathrm{Cm}^{\mathrm{R}}\right)$ & Invitrogen \\
\hline W3110 & $\mathrm{F}^{-}, \lambda^{-} \mathrm{IN}(r r n D-r r n E) 1 r p h-1$ & Laboratory collection \\
\hline
\end{tabular}

\section{PLASMIDS}

\begin{tabular}{|c|c|c|}
\hline pETG20A & $\mathrm{Amp}^{\mathrm{R}}$, ColE1, pT7, Gateway ${ }^{\circledR}$ destination vector, $6 \times$ His-TRX followed by a TEV cleavage site & Arie Gerlof \\
\hline pET-VgrG1- $\Delta$ gp5 & EAEC sci-1 $\operatorname{vgr} G(1-490)$ cloned upstream the TEV cleavage site into pETG20A & This study \\
\hline pET-VgrG2- $\triangle \mathrm{gp} 5$ & EAEC sci-2 vgrG (1-465) cloned upstream the TEV cleavage site into pETG20A & This study \\
\hline $\mathrm{pET}-\mathrm{VgrG}_{\mathrm{Vc}}-\Delta \mathrm{gp} 5$ & Vibrio cholerae $0395 \operatorname{vgrG}(1-471)$ cloned upstream the TEV cleavage site into pETG20A & This study \\
\hline
\end{tabular}




\begin{tabular}{|c|c|c|}
\hline pDEST14 & $\mathrm{Amp}^{\mathrm{R}}, \mathrm{pBR} 322$ ori, $\mathrm{pT} 7$, Gateway ${ }^{\circledR}$ destination vector, $6 \times$ His tag at $\mathrm{C}$-terminus & Thermofisher \\
\hline pDEST14-Hcp1 & EAEC $s c i-1$ hcp cloned into pDEST14 & Douzi et al., 2014 \\
\hline pDEST14-Hcp2 & EAEC sci-2 hcp cloned into pDEST14 & Douzi et al., 2014 \\
\hline pDEST14-Hcp $\mathrm{Vc}_{\mathrm{c}}$ & Vibrio cholerae $\mathrm{O} 395$ hcp cloned into pDEST14 & This study \\
\hline pEB354 & $\mathrm{Kan}^{\mathrm{R}}$, pKT25 derivative, P15A, Plac, T25 domain of Bordetella adenylate cyclase & Battesti \& Bouveret, 2006 \\
\hline pEB355 & $\mathrm{Amp}^{\mathrm{R}}$, pUT18 derivative, ColE1, Plac, T18 domain of Bordetella adenylate cyclase & Battesti \& Bouveret, 2006 \\
\hline pT18-Pal & pal cloned downstream T18 into pEB355 & Battesti \& Bouveret, 2006 \\
\hline pT25-TolB & tolB cloned downstream T25 into pEB354 & Battesti \& Bouveret, 2006 \\
\hline pT25-Hcp 1 & sci-1 hcp cloned downstream T25 into pEB354 & Zoued et al., 2013 \\
\hline pHcp1-T25 & sci-1 hcp cloned upstream T25 into pEB354 & Zoued et al., 2013 \\
\hline pT25-Hcp1-N93W & Asn93-to-Trp substitution introduced into pT25-Hcp1 & Brunet et al., 2014 \\
\hline pT25-Hcp1-S158W & Ser158-to-Trp substitution introduced into pT25-Hcp1 & Brunet et al., 2014 \\
\hline pVgrG1-T18 & sci-1 $\operatorname{vgr} G$ cloned upstream T18 into pEB355 & Zoued et al., 2013 \\
\hline pT18-TssA & sci-1 tss $A$ cloned downstream T18 into pEB355 & Zoued et al., 2013 \\
\hline pT25-VgrG1 & sci-1 vgrG cloned downstream T25 into pEB354 & Zoued et al., 2013 \\
\hline pT25-VgrG1loop & Ser360-to-Gly, Arg361-to-Gly and Lys362-to-Gly substitution introduced into pT25-VgrG1 & This study \\
\hline pBAD33 & $\mathrm{Cm}^{\mathrm{R}}, \mathrm{P} 15 \mathrm{~A}, \mathrm{pAraBAD}, \mathrm{AraC}$, cloning vector. & Guzman et al., 1995 \\
\hline pBAD-VgrG1 & sci-1 $\operatorname{vgr} G$ fused to a C-terminal VSV-G tag cloned into pBAD33 & This study \\
\hline pBAD-VgrG1- $\Delta$ DUF & sci-1 $\operatorname{vgr} G(1-573)$ fused to a C-terminal VSV-G tag cloned into pBAD33 & This study \\
\hline pBAD-VgrG1- $\Delta g \mathrm{~g} 5$ & sci-1 $\operatorname{vgrG}(1-490)$ fused to a C-terminal VSV-G tag cloned into pBAD33 & This study \\
\hline pBAD-VgrG1- $\triangle \mathrm{OB}$ & sci-1 $\operatorname{vgrG}(1-386)$ fused to a C-terminal VSV-G tag cloned into pBAD33 & This study \\
\hline pBAD-VgrG1-R361C & Arg361-to-Cys substitution introduced into pBAD-VgrG1 & This study \\
\hline pBAD-VgrG1-K362C & Lys362-to-Cys substitution introduced into pBAD-VgrG1 & This study \\
\hline pBAD-VgrG1loop & Ser360-to-Gly, Arg361-to-Gly and Lys362-to-Gly substitution introduced into pBAD-VgrG1 & This study \\
\hline pUC12 & $\mathrm{Amp}^{\mathrm{R}}$, ColE1, pLac, cloning vector & This study \\
\hline pUC12-Hcp1 & sci-1 hcp fused to a C-terminal FLAG tag cloned into pUC12 & Aschtgen et al., 2008 \\
\hline pUC12-Hcp2 & $s c i-2$ hcp fused to a C-terminal FLAG tag cloned into pUC12 & Aschtgen et al., 2008 \\
\hline pUC12-Hcp1-Q24C & Gln24-to-Cys substitution introduced into pUC12-Hcp1 & Brunet et al., 2014 \\
\hline pUC12-Hcp1-N46C & Asn46-to-Cys substitution introduced into pUC12-Hcp 1 & This study \\
\hline pUC12-Hcp1-G48C & Gly48-to-Cys substitution introduced into pUC12-Hcp1 & Brunet et al., 2014 \\
\hline pUC12-Hcp1-A95C & Ala95-to-Cys substitution introduced into pUC12-Hcp1 & Brunet et al., 2014 \\
\hline pUC12-Hcp1-G96C & Gly96-to-Cys substitution introduced into pUC12-Hcp1 & Brunet et al., 2014 \\
\hline pUC12-Hcp1-S158C & Ser158-to-Cys substitution introduced into pUC12-Hcp1 & Brunet et al., 2014 \\
\hline pUC12-Hcp1-Q24C-A95C & Ala95-to-Cys substitution introduced into pUC12-Hcp1-Q24C & Brunet et al., 2014 \\
\hline pUC12-Hcp1-G96C-S158C & Gly96-to-Cys substitution introduced into pUC12-Hcp1-S158C & Brunet et al., 2014 \\
\hline
\end{tabular}




\section{OLIGONUCLEOTIDES}

Insertion into pETG20A (Gateway cloning) ${ }^{\text {a, b }}$

\begin{tabular}{|c|c|}
\hline VgrG1-Fwd & $\begin{array}{l}\text { GGGGACAAGTTTGTACAAAAAAGCAGGCTTACACCACCACCACCACCACGAAAACCTGTACTTCCAGGGTATGAATCT } \\
\text { CACTGACTCCCTGC }\end{array}$ \\
\hline VgrG1- $\Delta$ gp5-Rev & GGACCACTTTGTACAAGAAAGCTGGGTCTTATTAGTTCTTATCGGTAACGTGATC \\
\hline VgrG2-Fwd & $\begin{array}{l}\text { GGGGACAAGTTTGTACAAAAAAGCAGGCTTACACCACCACCACCACCACGAAAACCTGTACTTCCAGGGTATGACTAG } \\
\text { GCAAAGATTTATATC }\end{array}$ \\
\hline VgrG2- $\Delta$ gp5-Rev & GGACCACTTTGTACAAGAAAGCTGGGTCTTATTAATTATTCTCAGGAAGAGAAAAAG \\
\hline $\begin{array}{l}\operatorname{VgrG}_{\mathrm{Vc}_{c}}-\mathrm{Fwd} \\
\mathrm{VgrGG}_{\mathrm{Vc}}-\Delta \mathrm{gp} 5-\mathrm{Rev}\end{array}$ & $\begin{array}{l}\text { ACAAAAAAGCAGGCTTACACCACCACCACCACCACGAAAACCTGTACTTCCAGGGTATGGCGACATTAGCGTACAGC } \\
\text { GGACCACTTTGTACAAGAAAGCTGGGTCTTATTAGTGCTGAGGCAGGTGTATGGCGG }\end{array}$ \\
\hline
\end{tabular}

Insertion into pDEST14 (Gateway cloning)

Hcpl-Fwd GGGGACAAGTTTGTACAAAAAAGCAGGCTTAGAAGGAGATAGAACCATGAAAGCAATTCCAGTTTATCTGTG Hcp1-Rev GGGGACCACTTTGTACAAGAAAGCTGGGTTTATTAATGGTGATGGTGATGGTGCGCGGTGGTACGCTCACTCC Hcp2-Fwd GGGGACAAGTTTGTACAAAAAAGCAGGCTTAGAAGGAGATAGAACCATGAAAGTCGGAGTTATGAGTAATTC Hcp2-Rev $\mathrm{Hcp}_{\mathrm{Vc}}-\mathrm{Fwd}$ GGGGACCACTTTGTACAAGAAAGCTGGGTTTATTAATGGTGATGGTGATGGTGTACAAGAGCCTCTTTATATAAG $\mathrm{Hcp}_{\mathrm{Vc}}-\mathrm{Rev}$ GGGGACAAGTTTGTACAAAAAAGCAGGCTTAGAAGGAGATAGAACCCCAACTCCATGTTATATCTCTATCG GGGGACCACTTTGTACAAGAAAGCTGGGTCTTAGTGATGGTGATGGTGATGCGCTTCGATTGGCTTACGCCAG

Insertion into pBAD33 (restriction-free cloning) ${ }^{\text {a, c, d }}$

5-pBAD-VgrG CTCTCTACTGTTTCTCCATACCCGTTTTTTTGGGCTAGCAGGAGGTATTACACCATGAATCTCACTGACTCCCTGCAAA $\frac{\text { ATGTTTTATCC }}{3-\mathrm{PBAD}-V \mathrm{grG}-573-\mathrm{CGTCGACTCTAGAGGAT}}$

3-pBAD-VgrG-573 GGTCGACTCTAGAGGATCCCCGGGTACCTTATTTTCCTAATCTATTCATTTCAATATCTGTATACTTTCCTTGTGCCTGAGGC TGCATATC

3-pBAD-VgrG-490 GGTCGACTCTAGAGGATCCCCGGGTACCTTATTTTCCTAATCTATTCATTTCAATATCTGTATAACCGTTCTTATCGGTAACG

$\frac{\text { TGATCAACATGAC }}{3-\mathrm{G}}$

3-pBAD-VgrG-386 GGTCGACTCTAGAGGATCCCCGGGTACCTTATTTTCCTAATCTATTCATTTCAATATCTGTATAAGTAAGTAACGGTGGTCGC CAGCAGAC 
Site-directed mutagenesis ${ }^{\mathrm{e}}$

$\begin{array}{ll}\text { A-Hcp1-N46C } & \text { TCTACATCCCGACGGATAACTGTACCGGTAAGCTGACCGG } \\ \text { B-Hcp1-N46C } & \text { CCGGTCAGCTTACCGGTACAGTTATCCGTCGGGATGTAGA } \\ \text { A-VgrG1-R361C } & \text { AGCTTCTGTTCACCGGTAGCTGTAAATCTGCGCTACAGGT } \\ \text { B-VgrG1-R361C } & \text { ACCTGTAGCGCAGATTTACAGCTACCGGTGAACAGAAGCT } \\ \text { A-VgrG1-K362C } & \text { TTCTGTTCACCGGTAGCCGGTGTTCTGCGCTACAGGTGAT } \\ \text { B-VgrG1-K362C } & \text { ATCACCTGTAGCGCAGAACACCGGCTACCGGTGAACAGAA } \\ \text { A-VgrG1-loop } & \text { GCAGCTTCTGTTCACCGGTGGCGGGGGATCTGCGCTACAGGTGATG } \\ \text { B-VgrG1-loop } & \text { CATCACCTGTAGCGCAGATCCCCCGCCACCGGTGAACAGAAGCTGC }\end{array}$

${ }^{a}$ sequence complementary to the pBAD33 plasmid (restriction-free cloning) or Gateway overhangs (Gateway cloning) underlined.

${ }^{\mathrm{b}} 6 \times$ His coding sequence in italics.

${ }^{c}$ RBS and ATG sequence in bold.

${ }^{\mathrm{d}}$ VSV-G tag coding sequence in italics.

${ }^{\mathrm{e}}$ mutagenesized codon underlined. 
Figure S1
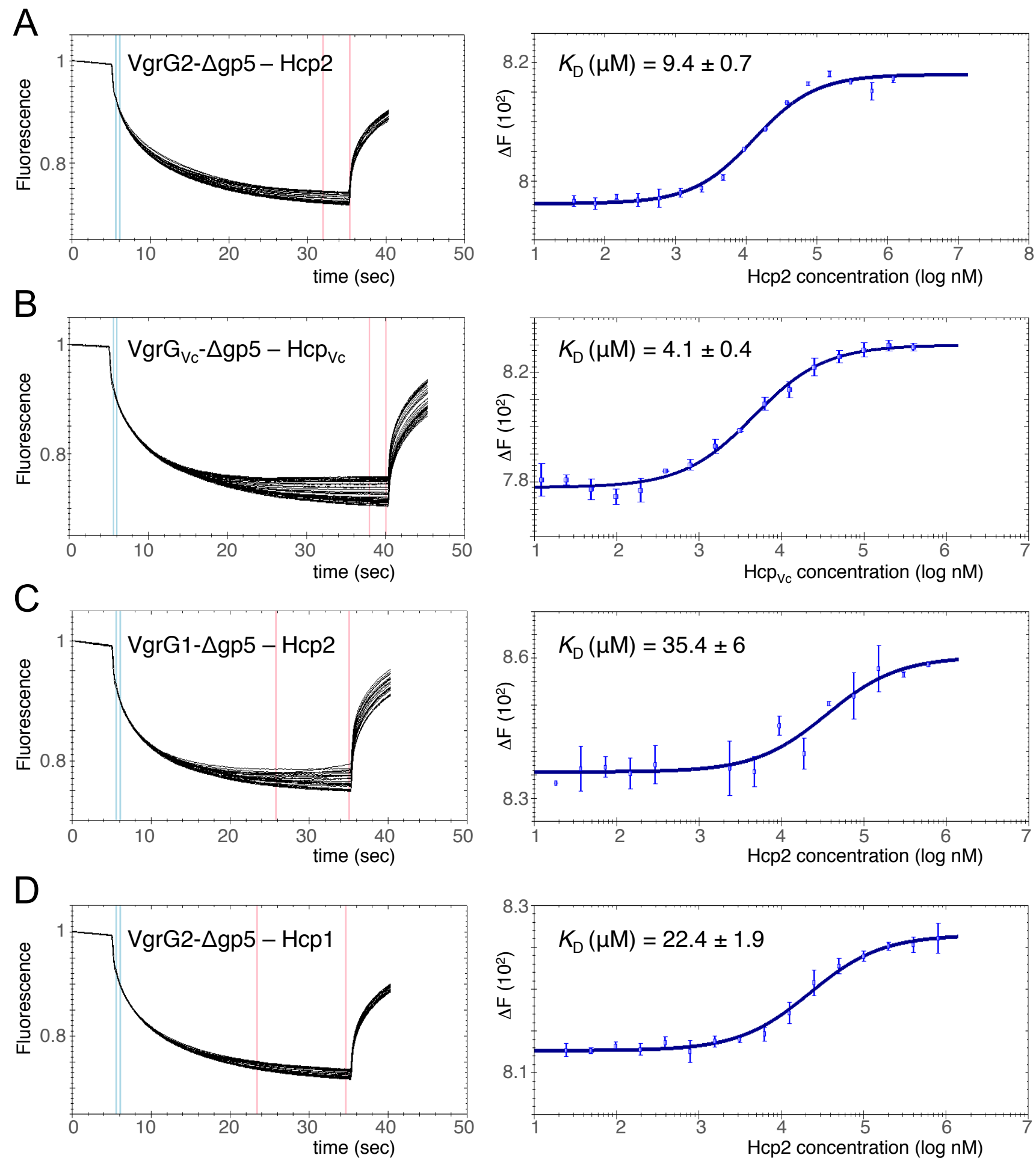

Figure S1. Microscale thermophoresis affinity measurements between cognate and non-cognate VgrG and Hcp proteins. Thermophoretic time trace recordings of the unlabeled EAEC Hcp1 or Hcp2, or V. cholerae Hcp (Hcp $\mathrm{Vc}_{\mathrm{c}}$ ) titration (from $0.9 \mathrm{nM}$ to 600 $\mu \mathrm{M}$ ) to a constant amount of fluorescently labeled EAEC VgrG1- $\Delta \mathrm{gp} 5$ or VgrG2- $\Delta \mathrm{gp} 5$ or $V$. cholerae $\mathrm{VgrG}_{\mathrm{Vc}}-\Delta \mathrm{gp} 5$ (left panels). The measured changes in the MST response $(\Delta \mathrm{F})$ were plotted against the Hcp concentration to calculate the binding constant (right panels). The VgrG2- $\Delta \mathrm{gp} 5$ - Hcp2 (panel A, $\left.\mathrm{K}_{D}=9.4 \pm 0.7 \mu \mathrm{M}\right), \operatorname{VgrG}_{\mathrm{Vc}_{\mathrm{c}}}-\Delta \mathrm{gp} 5-\mathrm{Hcp}_{\mathrm{Vc}_{\mathrm{c}}}$ (panel B, $\left.\mathrm{K}_{D}=4.1 \pm 0.4 \mu \mathrm{M}\right), \mathrm{VgrG} 1-\Delta \mathrm{gp} 5-$ Hcp2 (panel C, $\mathrm{K}_{D}=35.4 \pm 6 \mu \mathrm{M}$ ) and $\mathrm{VgrG} 2-\Delta \mathrm{gp} 5$ - Hcp1 (panel D, $\mathrm{K}_{D}=22.4 \pm 1.9 \mu \mathrm{M}$ ) recordings are shown. 


\section{Figure S2}

A

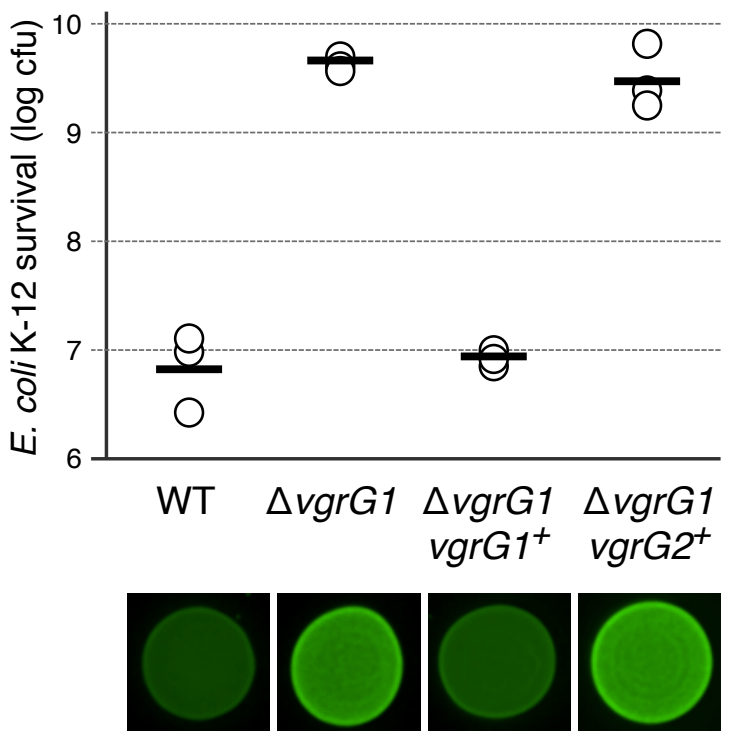

B

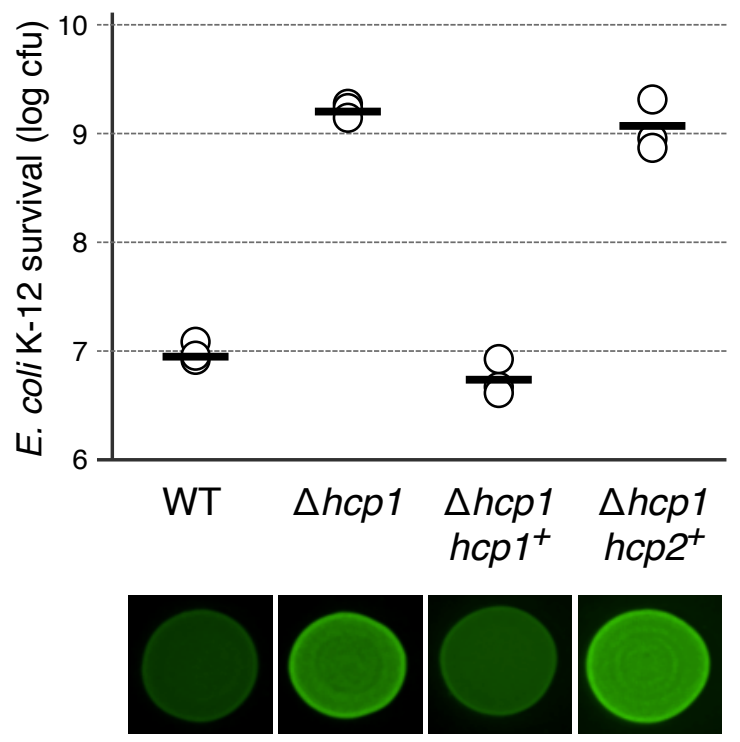

Figure S2. VgrG2 and Hcp2 do not compensate the absence of VgrG1 and Hcp1 for Sci1 T6SS-mediated inter-bacterial activity. E. coli K-12 recipient cells (W3110 $g f p^{+}$, $\mathrm{kan}^{\mathrm{R}}$ ) were mixed with the indicated attacker cells: WT, EAEC 17-2; $\Delta v g r G 1$, $\Delta v g r G 1$ cells carrying the pBAD33 empty vector; $v g r G 1^{+}, \Delta v g r G 1$ cells producing VgrG1; vgrG2 $2^{+}, \Delta v g r G 1$ cells producing $\operatorname{VgrG} 2$ (panel A); WT, EAEC 17-2; $\Delta h c p 1, \Delta h c p 1$ cells carrying the pUC12 empty vector; $h c p 1^{+}, \Delta h c p 1$ cells producing Hcp1; $h c p 2^{+}, \Delta h c p 1$ cells producing Hcp2 (panel B). The mixtures were spotted onto Sci-1 inducing medium (SIM) agar plates and incubated for 4 hours at $37^{\circ} \mathrm{C}$. The image of a representative bacterial spot is shown below the graph reporting the number of surviving $E$. coli prey cells (counted on selective kanamycin medium; in $\log 10$ of colony-forming units (cfu)). The open circles indicate values from three independent assays, and the average is indicated by the bar. 


\section{Figure S3}

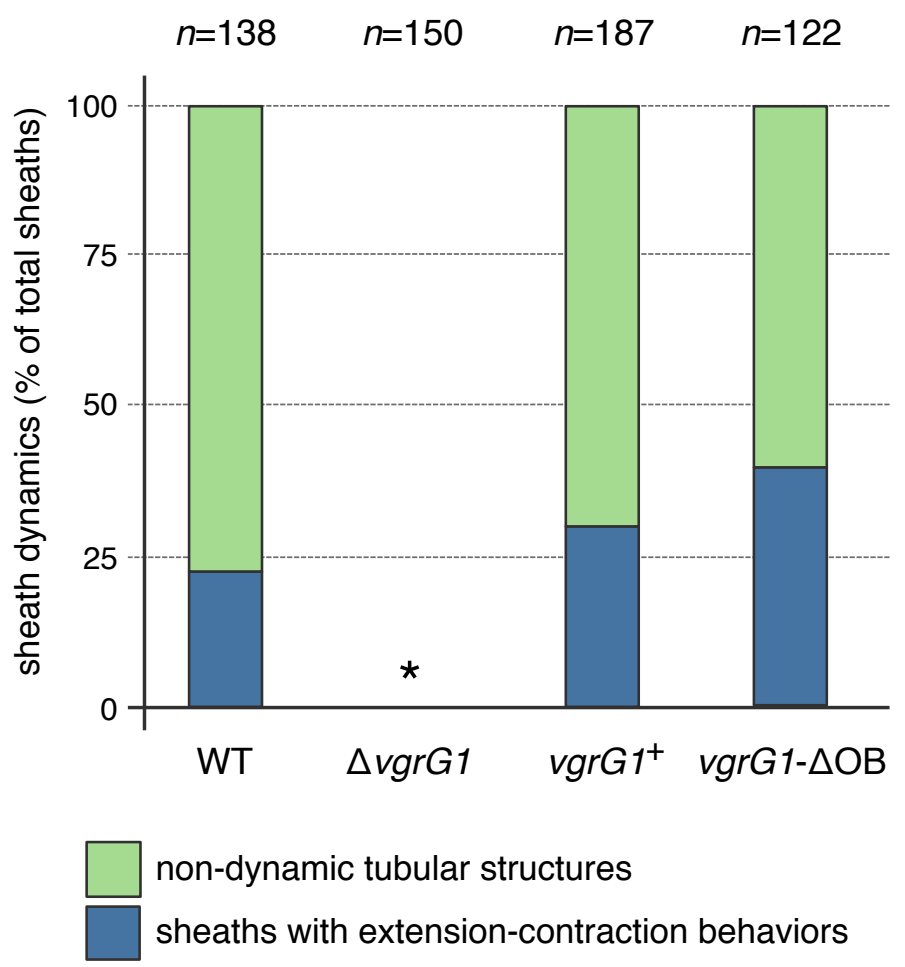

Figure S3. Statistical analyses of sheaths behavior. Distribution of the dynamic behavior of T6SS sheaths, monitored by time-lapse fluorescence recordings of the indicated strain (WT, EAEC 17-2; $\Delta v \operatorname{vg} G 1, \Delta \operatorname{vgr} G 1$ cells carrying the pBAD33 empty vector; $v g r G 1^{+}$,

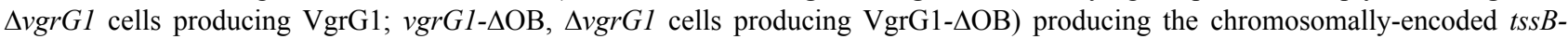
$m$ Cherry fusion. The bars represent the percentage of non-dynamic tubular structures (blue) and sheaths that undergo cycles of extension-contraction (green). The number of cells analysed $(n)$ is indicated on top. The asterisk $(*)$ indicates that no sheath is observable in this strain (diffuse fluorescence). 


\section{Figure S4}

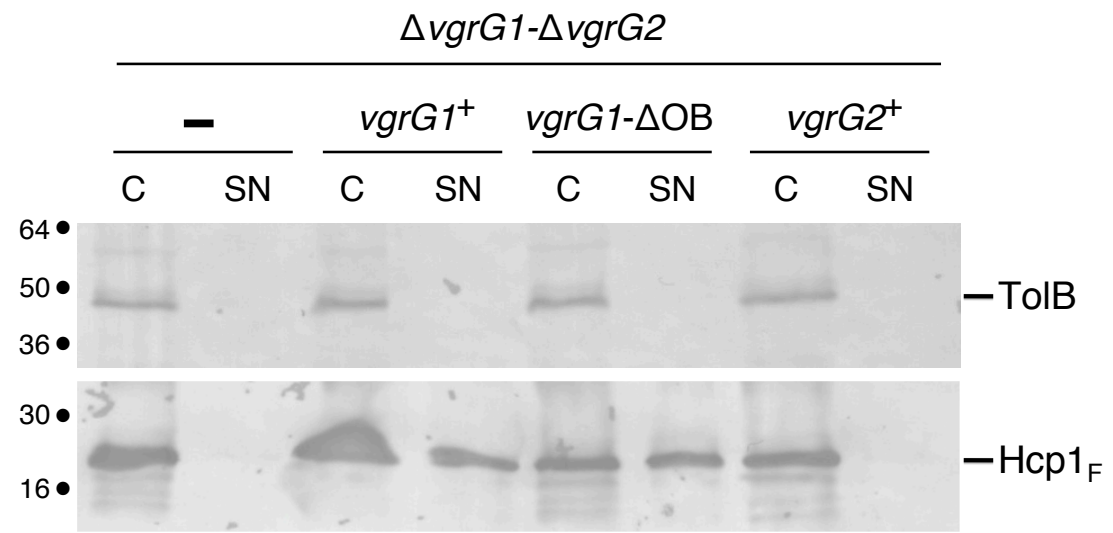

Figure S4. The gp27-like base of VgrG1 does not require VgrG2 to promote Hcp1 release. FLAG-tagged Hcp1 (Hcp1 $\left.{ }_{\mathrm{F}}\right)$ release was assessed by separating total cell (C) and cell-free culture supernatant (SN) fractions from $5 \times 10^{8} \Delta v g r G 1-\Delta v g r G 2$ cells carrying the empty pBAD33 vector (-), the pBAD33 vector producing VgrG1 $\left(v g r G 1^{+}\right)$, $\operatorname{VgrG1}-\Delta \mathrm{OB}(\operatorname{vgr} G 1-\Delta \mathrm{OB})$ or $\operatorname{VgrG} 2\left(\operatorname{vgrG} 2^{+}\right)$. Proteins were separated by $12.5 \%$-acrylamide SDS-PAGE and periplasmic TolB and $\mathrm{Hcp}_{\mathrm{F}}$ were immunodetected using anti-TolB (upper panel) and anti-FLAG (lower panel) antibodies. Molecular weight markers (in kDa) are indicated on the left. 


\section{Figure S5}

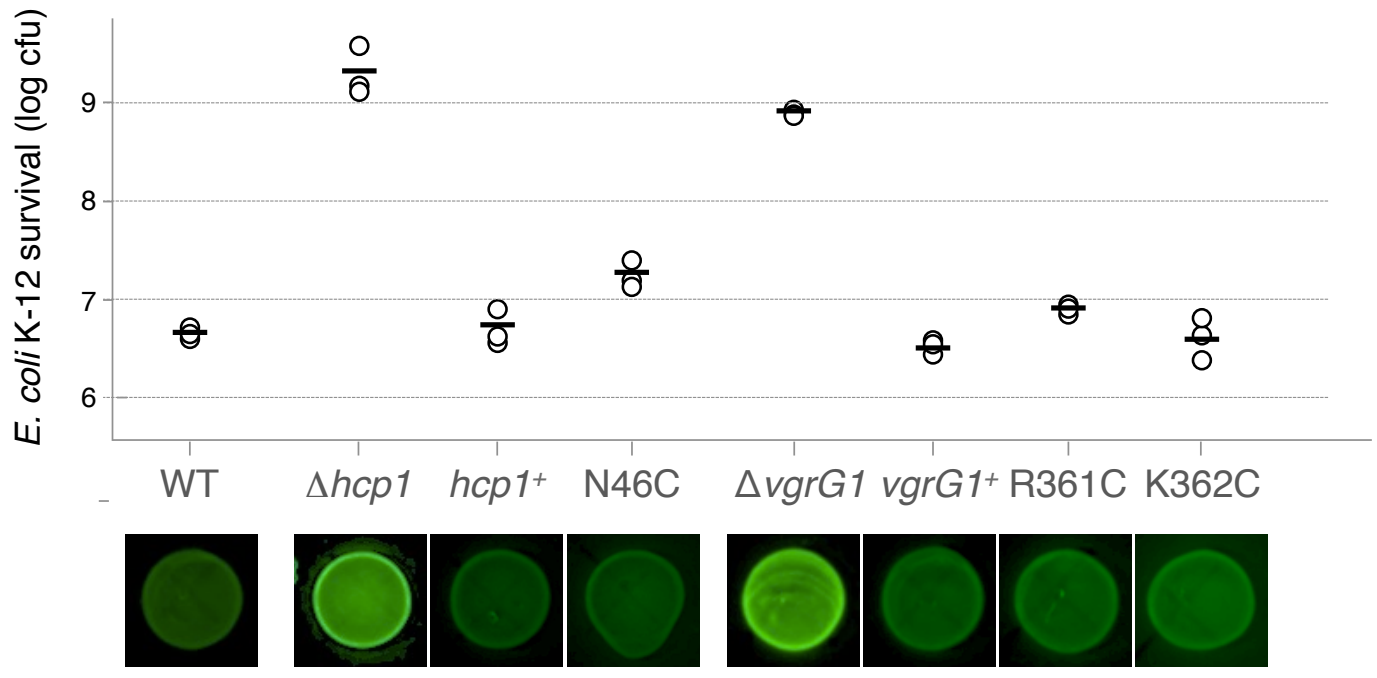

Figure S5. Hep1 and VgrG1 cysteine variants are functional for T6SS-dependent inter-bacterial activity. E. coli K-12 recipient cells (W3110 $g f p^{+}, \operatorname{kan}^{\mathrm{R}}$ ) were mixed with the indicated attacker cells (WT, EAEC 17-2; $\Delta h c p l, \Delta h c p 1$ cells carrying the pUC12 empty vector; $h c p 1^{+}, \Delta h c p 1$ cells producing Hcp1; N46C, $\Delta h c p 1$ cells producing the Hcp1 N46C variant; $\Delta v g r G 1, \Delta v g r G 1$ cells carrying the pBAD33 empty vector; $v g r G 1^{+}, \Delta v g r G 1$ cells producing $\operatorname{VgrG} 1$; R361C and K362C, $\Delta v g r G 1$ cells producing the VgrG1 $\mathrm{R} 361 \mathrm{C}$ and $\mathrm{K} 362 \mathrm{C}$ variants, respectively), spotted onto Sci-1 inducing medium (SIM) agar plates and incubated for 4 hours at $37^{\circ} \mathrm{C}$. The image of a representative bacterial spot is shown below the graph reporting the number of surviving $E$. coli prey cells (counted on selective kanamycin medium; in $\log 10$ of colony-forming units (cfu)). The open circles indicate values from three independent assays, and the average is indicated by the bar. 